\title{
Microscopic description of cluster radioactivity in actinide nuclei
}

\author{
M. Warda* \\ Katedra Fizyki Teoretycznej, Uniwersytet Marii Curie-Skłodowskiej, ul. Radziszewskiego 10, PL-20-031 Lublin, Poland \\ L. M. Robledo ${ }^{\dagger}$ \\ Departamento de Física Teórica (Módulo 15), Universidad Autónoma de Madrid, E-28049 Madrid, Spain
}

(Received 5 July 2011; revised manuscript received 10 August 2011; published 13 October 2011)

\begin{abstract}
Cluster radioactivity is the emission of a fragment heavier than an $\alpha$ particle and lighter than mass 50 . The range of clusters observed in experiments goes from ${ }^{14} \mathrm{C}$ to ${ }^{32} \mathrm{Si}$ while the heavy mass residue is always a nucleus in the neighborhood of the doubly-magic ${ }^{208} \mathrm{~Pb}$ nucleus. Cluster radioactivity is described in this paper as very asymmetric nuclear fission. A new fission valley leading to a decay with large fragment mass asymmetry matching the cluster radioactivity products is found. The mass octupole moment is found to be more convenient than the standard quadrupole moment as the parameter driving the system to fission. The mean-field Hartree-Fock-Bogoliubov theory with the phenomenological Gogny interaction has been used to compute the cluster emission properties of a wide range of even-even actinide nuclei from ${ }^{222} \mathrm{Ra}$ to ${ }^{242} \mathrm{Cm}$, where emission of the clusters has been experimentally observed. Computed half-lives for cluster emission are compared with experimental results. The noticeable agreement obtained between the predicted properties of cluster emission (namely, cluster masses and emission half-lives) and the measured data confirms the validity of the proposed methodology in the analysis of the phenomenon of cluster radioactivity. A continuous fission path through the scission point has been described using the neck parameter constraint.
\end{abstract}

DOI: 10.1103/PhysRevC.84.044608

PACS number(s): 23.70.+j, 25.85.Ca, 27.90.+b, 21.60.Jz

\section{INTRODUCTION}

The emission of $\alpha$ particles and nuclear fission are the two dominant spontaneous decay modes of heavy and superheavy nuclei. In both cases two nuclei are produced. In $\alpha$ decay one ${ }^{4} \mathrm{He}$ nucleus ( $\alpha$ particle) is emitted out of the parent nucleus and the remaining nucleons form a heavy mass residue with $N-2$ neutrons and $Z-2$ protons. In contrast to the huge mass asymmetry of $\alpha$ decay, two nuclei of similar mass are created in nuclear fission. A large variety of isotopes are produced in spontaneous fission with masses covering the range from $A \sim 70$ to $A \sim 190$. In many heavy nuclei the dominant decay channel corresponds to asymmetric fission with the most probable mass of heavy fragment $A \sim 140$ and the mass of the light one in the range from $A \sim 100$ to $A \sim 120$ depending on the mass of parent nucleus. Symmetric fission is also possible in some nuclei with the most probable division into two similar fragments. Daughter nuclei lighter than $A \sim 60$ have never been observed in any fission experiment. Therefore there is a clear distinction between $\alpha$ emission and fission regarding the mass of the lighter products as it shows a gap of light nuclei with $A \sim 10-50$ that cannot be produced in either of the two mentioned decay channels of any heavy nuclei. The source of the observed differences can be easily explained from basic nuclear properties, namely, the energetic balance of the two reactions. Fission is favorable energetically because of the linear decreasing behavior of the binding energy per nucleon for mass numbers larger than $A \sim 60$ (the iron peak) that prevents fragments with mass numbers lower than that

\footnotetext{
*warda@kft.umcs.lublin.pl
}

†luis.robledo@uam.es value. On the other hand, $\alpha$ decay energetics is dominated by the huge binding energy (as compared to neighboring nuclei) of the $\alpha$ particle.

A common aspect of fission and $\alpha$ decay is that the dynamical evolution from the parent nucleus to the daughter is not favorable energetically, although the $Q$ value of both reactions is positive. Therefore the quantum mechanics mechanism of tunneling through a potential barrier is required to explain both types of decay. As tunneling probabilities depend exponentially on the width and height of the barrier the expected half-lives can span a wide range of many orders of magnitude. This peculiarity makes understanding fission and $\alpha$ decay very challenging.

In 1984 Rose and Jones [1] observed for the first time the emission of a ${ }^{14} \mathrm{C}$ nucleus from a ${ }^{223} \mathrm{Ra}$ probe. This discovery represented a milestone in the description of nuclear radioactivity as it bridged the gap between the $\alpha$ emission radioactivity and the standard fission reaction. Since then, cluster radioactivity (CR) has been found in twelve eveneven isotopes [2-21] and seven odd-even isotopes (see, e.g., references in Refs. [22,23]) in the actinide region. They range from ${ }^{221} \mathrm{Fr}$ up to ${ }^{242} \mathrm{Cm}$. The emission of ${ }^{14} \mathrm{C},{ }^{20} \mathrm{O},{ }^{23} \mathrm{~F},{ }^{24-26} \mathrm{Ne}$, ${ }^{28-30} \mathrm{Mg}$, and ${ }^{32,34} \mathrm{Si}$ has been observed. The common factor of all cluster radioactivity events is the heavy-mass residue which is in the neighborhood of the doubly-magic ${ }^{208} \mathrm{~Pb}$. This fact allows us to better characterize CR as "lead radioactivity" and indicates the strong influence of shell effects on the nature of this phenomenon.

Experiments aiming to find $\mathrm{CR}$ in the distant region of the neutron-deficient $\mathrm{Ba}$ isotopes have been described in Refs. [24-26]. In this case another doubly-magic nucleus, namely, ${ }^{100} \mathrm{Sn}$, can be considered as the heavy residue and the carbon isotopes around ${ }^{12} \mathrm{C}$ are expected to be emitted. The 
experiments did not provide evidence for $\mathrm{CR}$ in this region and quantitatively they only gave lower limits for the branching ratios for ${ }^{12} \mathrm{C}$ emission.

Cluster radioactivity is an exotic process. The partial half-lives are very long and vary in a wide range from $10^{11}$ to $10^{26} \mathrm{~s}$. Branching ratios to the dominant $\alpha$ decay in these nuclei are very small and fall between $10^{-9}$ and $10^{-16}$. Moreover, spontaneous fission is also a competing decay channel in some heavy cluster emitters [27]. These reasons clearly justify why CR was experimentally discovered as late as 45 years after the first fission events, which were reported back in 1939 [28]. In the last few decades and thanks to both the interest raised by the phenomenon and the impressive improvement of experimental techniques, many examples of CR have been found in several actinide nuclei. Various experimental methods have been applied to detect the products of cluster emission [22,23,29]. Initial observations were based on techniques borrowed from $\alpha$ decay studies. A $\Delta E-E$ telescope made of silicon detectors was used by Rose and Jones in the first experiment [1]. This method was inconvenient due to the huge $\alpha$ radioactivity background, which could even destroy the experimental setup. Later, a magnetic field was applied to remove the background of charged $\alpha$ particles. Another method used in experiments was the detection of $\gamma$ rays emitted from exited clusters. Numerous clusters were identified in solid-state nuclear track detectors. In this technique plastic or glass layers absorb the ionized cluster emitted from the radioactive probe. The material of the layer cannot be sensitive to $\alpha$ radiation and plastic or glass materials with proper ionization thresholds are the standard choices. After irradiation, the layer is etched to enlarge the track created by the emitted cluster and make it visible and well defined under the microscope. The analysis of the geometry of the track allows one to identify the emitted cluster.

On the theoretical side, the first successful theoretical description of cluster decay was made by Sandulescu et al. [30], four years before the experimental discovery of this reaction. Since the pioneering work of Sandulescu, numerous theoretical papers devoted to this end have been published [31-59]. A thorough overview of most of the theoretical (mostly semimicroscopic) methods can be found in Refs. [23,60-63].

As CR is a decay mode "in between" $\alpha$ emission and nuclear fission, methods already known to both of them can be used to describe cluster radioactivity. For instance, the Gamow model of $\alpha$ emission can be extrapolated to describe the emission of heavier clusters. It requires the assumption that a cluster of nucleons is preformed in the interior of parent nucleus and then it tunnels through the barrier of nuclear and Coulomb potential. In this way a kind of universal decay law similar to the Geiger-Nuttall formula for $\alpha$ emission can be formulated $[23,52,57]$. The main drawback of this approach is that the preformation of the cluster inside the parent nucleus is a poorly known and hard to characterize process [64-66]. Nevertheless, the half-lives predicted by this method agree very well with the experimental data.

The other method treats cluster radioactivity as a very asymmetric fission reaction (see, e.g., Refs. [30,60,62,63]). The formation of the cluster is a direct consequence of a specific kind of deformation of the parent nucleus. In this approach a fission barrier with a specific mass division must be determined. The locally maximal barrier transition probability for the specific fragments with large mass asymmetry points to the possibility of fission with a cluster as one of the fragments. Usually the potential energy surface (PES) has to be determined as a function of the relevant deformation parameters, including elongation and reflection asymmetry coordinates. The path in this multidimensional deformation surface leading to fission with large fragment mass asymmetry has to be found and, finally, the fission barrier must be specified. In this approach it is reasonable to use two-center models in the description of the nuclear potential $[67,68]$.

We want to show that CR can be fully described microscopically as a very asymmetric fission process. We apply standard methods used in the theoretical description of nuclear fission which are well established in the literature [69-77]. We use the mean-field approximation in the Hartree-Fock-Bogoliubov (HFB) scheme with the finite-range Gogny force [78] to compute the nuclear wave functions. Axial symmetry of the nuclear system is assumed throughout the calculations. Constraints on the quadrupole and octupole moments allow us to control simultaneously the elongation and reflection asymmetry of the system as it evolves to the scission point. The wave functions obtained in this way can be used to calculate the necessary quantities (energies, collective masses, etc.) for a physical description of the process. Moreover, an extra constraint on the number of nucleons in the neck (the neck thickness) has been used to control the density distribution around the scission point. A description of CR is possible owing to the identification of a new valley in the PES leading to hyper-asymmetric fission. Charge and mass numbers of the light fragment created at the hyper-asymmetric scission point correspond to what is observed experimentally for a given nucleus. In contrast to the standard fission path where the leading coordinate is the quadrupole moment, in the hyper-asymmetric fission path the relevant coordinate turns out to be the octupole moment. Therefore, in our description of $\mathrm{CR}$, all physical quantities will be given in terms of the octupole moment.

The first results obtained in this approach have been published in the previous papers [31-34]. Cluster radioactivity of selected nuclei have been discussed there with some approximations of the model.

In this paper we want to investigate from a microscopic perspective all even-even actinide nuclei where cluster radioactivity has been experimentally detected. There are twelve such isotopes: ${ }^{222,224,226} \mathrm{Ra},{ }^{228,230} \mathrm{Th},{ }^{230,232,234,236} \mathrm{U},{ }^{236,238} \mathrm{Pu}$, and ${ }^{242} \mathrm{Cm}$. Moreover, three other nuclei $\left({ }^{226,232} \mathrm{Th},{ }^{240} \mathrm{Pu}\right)$, where experiments have only provided lower limits for half-lives of $\mathrm{CR}$, have been examined.

The structure of the paper is as follows: In Sec. II the theoretical model used in this investigation is described in detail. Two typical and representative examples of cluster radioactivity corresponding to the parent nuclei ${ }^{224} \mathrm{Ra}$ and ${ }^{238} \mathrm{Pu}$ are thoroughly discussed in Sec. III to establish the relevant physics driving the cluster emission process. Results for all fifteen nuclei considered in this paper are presented in Sec. IV. We conclude in Sec. V with the main consequences extracted from our theoretical description. 


\section{THEORY}

As a first step in our theoretical description of cluster emission we solve the mean-field HFB equation [79] with the usual constraints on the average number of particles and, in the present case, with a constraint on the value of the mass multipole moments $\left\langle\hat{Q}_{\lambda 0}\right\rangle=Q_{\lambda}$ to analyze the physical contents of the process. The axial quadrupole $\left(Q_{2}\right)$, octupole $\left(Q_{3}\right)$, and hexadecapole $\left(Q_{4}\right)$ moments are defined through the standard Legendre polynomials

$$
\hat{Q}_{\lambda 0}=r^{\lambda} P_{\lambda}[\cos (\theta)] .
$$

The nonlinear HFB equation is solved using the gradient method [80] and taking into account approximately secondorder curvature effects $[69,81]$. The HFB quasiparticle creation and annihilation operators are expanded in a harmonic oscillator (HO) basis and special attention is paid to the convergence of the results with the basis size (see Appendix A for further details). The interaction used is the finite-range Gogny force with the D1S parametrization [73]. This interaction has been proven to successfully describe the fission process in heavy nuclei $[69,74,76,77,82-87]$. The other Gogny forces developed recently_D1N [88] and D1M [89]—are discussed in Appendix B. Other details of the HFB calculations are as follows: the two-body kinetic energy correction (2bKEC) has been included in the minimization process. The exchange Coulomb contribution is evaluated in the Slater approximation.

All calculations have been performed in the axially symmetric regime. This seems to be a rational choice as the systems studied tend to be built from a large spherical part reproducing properties of doubly-magic nuclei with a small additional part. The lighter fragment is often spherical in the ground state. Therefore, the influence of nonaxial effects is expected to be rather small, if any, and may only affect the shape of the barrier just before scission, slightly reducing its height.

To evaluate the PES we take into account correlation energies beyond the mean field. To this end we subtract from the HFB energy the rotational energy corrections (RECs) stemming from the restoration of rotational symmetry. This correction has a considerable influence on the energy landscape (and therefore on the height of fission barriers) as it is proportional to the degree of rotational symmetry breaking. A full calculation of the REC would imply the evaluation of the angular momentum projected energy [90,91]. Unfortunately, this kind of beyond-mean-field calculation is only feasible for light nuclei with present-day computer capabilities. In order to estimate the REC we have followed the usual recipe [79] (which is well justified for strongly deformed configurations) of subtracting from the HFB energy the quantity $\left\langle\Delta \vec{J}^{2}\right\rangle /\left(2 \mathcal{J}_{Y}\right)$, where $\left\langle\Delta \vec{J}^{2}\right\rangle$ is the fluctuation on angular momentum of the HFB wave function and $\mathcal{J}_{Y}$ is the Yoccoz moment of inertia [92]. This moment of inertia has been computed using the "cranking" approximation in which the full linear response matrix appearing in its expression is replaced by the zero-order approximation (that is, the sum of two quasiparticle energies). The impact of this approximation on the value of the Yoccoz moment of inertia was analyzed with the Gogny interaction for heavy nuclei in [86] by comparing the approximate value with the one extracted from a complete angular momentum projected calculation (see also [90] for a comparison in light nuclei). The conclusion is that, for strongly deformed configurations, the exact REC is roughly a factor 0.7 smaller than the one computed with the "cranking" approximation to the Yoccoz moment of inertia. It has also to be mentioned that a similar behavior has been observed for the differences between the Thouless-Valatin moment of inertia computed exactly and that in the "cranking" approximation $[93,94]$. We have taken this phenomenological factor into account in our calculation of the REC.

In Sec. IV we will discuss half-lives corresponding to cluster emission and compare them with experimental data. The half-lives for cluster emission are computed (in seconds) using the standard WKB framework [95]

$$
t_{1 / 2}=2.86 \times 10^{-21}[1+\exp (2 S)] .
$$

The quantity $S$ entering this expression is the action along the $Q_{3}$ constrained path:

$$
S=\int_{a}^{b} d Q_{3} \sqrt{2 B\left(Q_{3}\right)\left[V\left(Q_{3}\right)-E_{0}\right]} .
$$

For the collective inertia $B\left(Q_{3}\right)$ we have used the adiabatic time-dependent HFB (ATDHFB) expression computed again in the "cranking" approximation and given by [94]

$$
B_{\text {ATDHFB }}\left(Q_{3}\right)=\frac{M_{-3}\left(Q_{3}\right)}{M_{-1}^{2}\left(Q_{3}\right)}
$$

with the moments $M_{-n}$ given by

$$
M_{-n}\left(Q_{3}\right)=\sum_{\mu \nu} \frac{\left|\left(Q_{30}^{20}\right)_{\mu \nu}\right|^{2}}{\left(E_{\mu}+E_{\nu}\right)^{n}} .
$$

In this expression, $\left(Q_{30}^{20}\right)_{\mu \nu}$ is the two-quasiparticle-zero-hole component of the octupole operator $\hat{Q}_{30}$ in the quasiparticle representation [79] and $E_{\mu}$ are the one-quasiparticle excitation energies obtained as the eigenvalues of the HFB Hamiltonian matrix.

In the expression for the action $V\left(Q_{3}\right)=E_{\mathrm{HFB}}\left(Q_{3}\right)-$ $\operatorname{REC}\left(Q_{3}\right)-\epsilon_{0}\left(Q_{3}\right)$ is given by the HFB energy minus the REC and the zero-point-energy (ZPE) correction $\epsilon_{0}\left(Q_{3}\right)$ associated with the octupole motion. This ZPE correction is given by

$$
\epsilon_{0}\left(Q_{3}\right)=\frac{1}{2} G\left(Q_{3}\right) B_{\mathrm{ATDHFB}}^{-1}\left(Q_{3}\right),
$$

where

$$
G\left(Q_{3}\right)=\frac{M_{-2}\left(Q_{3}\right)}{2 M_{-1}^{2}\left(Q_{3}\right)} .
$$

In the expression for the action an additional parameter $E_{0}$ is introduced. This parameter can be taken as the HFB energy of the (metastable) ground state. However, it is argued that in a quantal treatment of the problem the ground-state energy is given by the HFB energy plus the ZPE associated with the collective motion. To account for this fact, the usual recipe is to add to the HFB energy an estimation of the ZPE for the ground state in order to obtain $E_{0}$. In our calculations we have considered this ZPE as a phenomenological parameter and given a reasonable value of $0.5 \mathrm{MeV}$ for all isotopes considered 
[96]. Notice that this constant quantity is not canceling out the impact of $\epsilon_{0}\left(Q_{3}\right)$ in $V\left(Q_{3}\right)$. Finally, the turning points $a$ and $b$ in the limits of the integral of Eq. (3) are the intersection points between the horizontal line at $E_{0}$ and the $V\left(Q_{3}\right)$ curve.

\section{CLUSTER RADIOACTIVITY IN ${ }^{224}$ Ra AND ${ }^{238} \mathbf{P u}$}

The analysis of CR requires the determination of the PES for each nuclei considered in this paper. After performing these calculations we have found that there are no substantial qualitative differences among the various actinide isotopes considered. In all cases the PES is similar and only quantitative variations are found. Therefore we will not describe in detail the PES of all actinides. In this section we will concentrate only on the CR of two representative nuclei, namely, the light cluster emitter ${ }^{224} \mathrm{Ra}$, in which emission of ${ }^{14} \mathrm{C}$ is observed [2,15], and one of the heaviest emitters ${ }^{238} \mathrm{Pu}$, that decays by producing the relatively large clusters ${ }^{28,30} \mathrm{Mg}$ and ${ }^{32} \mathrm{Si}$ [7]. A detailed account of our previous calculations in some other isotopes can be found in [31-34].

\section{A. The PES and shapes of fissioning nuclei}

In Fig. 1 we show the PES of ${ }^{224} \mathrm{Ra}$ and ${ }^{238} \mathrm{Pu}$ in the deformation space of the quadrupole $Q_{2}$ and octupole $Q_{3}$ moments. This figure shows how the energy of the system changes with the simultaneous changes of elongation (controlled by $Q_{2}$ ) and reflection asymmetry (governed by $Q_{3}$ ). Calculations have been performed on a grid, with a spacing of $5 \mathrm{~b}$ in the $Q_{2}$ direction and of $5 \mathrm{~b}^{3 / 2}$ in the $Q_{3}$ direction. The oscillator lengths characterizing the single-particle basis have been optimized in every mesh point to minimize the total HFB energy. All the values of potential energies presented in this paper are the corresponding HFB energies corrected by the correlation energies of $2 \mathrm{bKEC}$ and REC as described in Sec. II. Both quantities represent correlation energies gained by restoring (in an approximate way) the rotational and translational symmetries spontaneously broken by the mean-field approximation. Moreover, to facilitate the analysis of the barriers heights, we have normalized energies to zero in the ground state.

In both nuclei the ground state is well deformed. Its quadrupole moment is $Q_{2}=8.3 \mathrm{~b}\left(\beta_{2}=0.18\right)$ for ${ }^{224} \mathrm{Ra}$ and $Q_{2}=14.1 \mathrm{~b}\left(\beta_{2}=0.27\right)$ for ${ }^{238} \mathrm{Pu}$. A small octupole deformation $Q_{3}=4.2 \mathrm{~b}^{3 / 2}\left(\beta_{3}=0.14\right)$ can also be found in the ground state of ${ }^{224} \mathrm{Ra}$. Fission valleys are characterized by a local decrease of the slope in the PES from the ground state toward scission. Fission paths can be found in the bottom of these valleys to determine locally the lowest energy barriers. The direction corresponding to the slowest increase of the potential energy with deformation can be easily found along the reflection symmetric axis. This barrier is also plotted in Fig. 2 with a green short-dashed line. At $Q_{2}=20-25$ b the barrier reaches a saddle point and then it slowly descends. At larger elongation, from $Q_{2}=50 \mathrm{~b}$, the potential energy increases again, producing a second hump of the barrier. At this stage, the fission valley turns into reflection-asymmetric shapes and a second saddle point can be found around
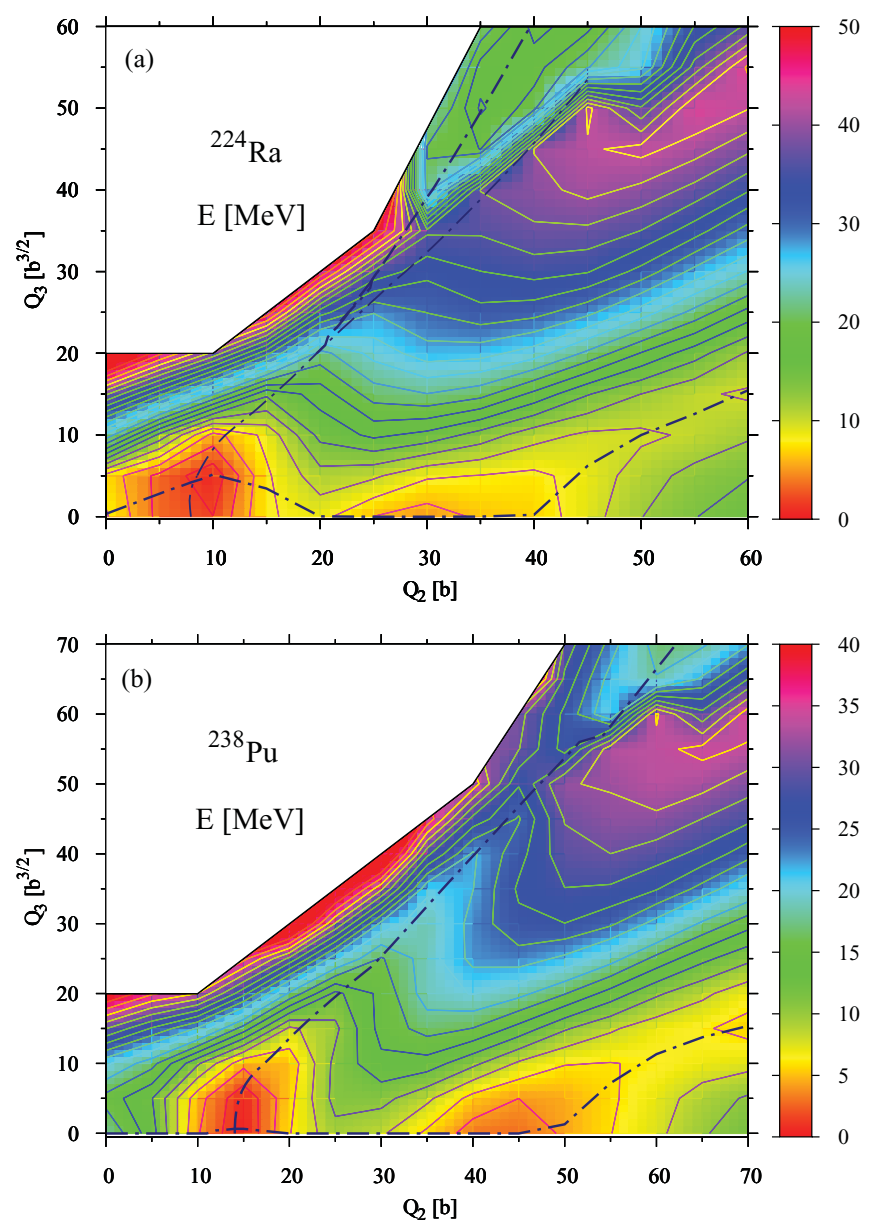

FIG. 1. (Color online) Maps of the PES of (a) ${ }^{224} \mathrm{Ra}$ and (b) ${ }^{238} \mathrm{Pu}$ as a function of quadrupole moment $Q_{2}$ and octupole moment $Q_{3}$. Lines of constant energy are plotted every $2 \mathrm{MeV}$. Bold dot-dashed lines are plotted along fission paths.

$Q_{2}=55-60 \mathrm{~b}$ with $Q_{3}=15 \mathrm{~b}^{3 / 2}$. This is the typical scenario of fission in many heavy nuclei leading to asymmetric fission. Such a valley is usually called an "elongated fission valley" $[69,70]$ as the shapes of the nucleus along it are relatively stretched with a long neck coupling a typically spherical fragment and a typically prolate-deformed nascent fragment. The value of the fission barrier height is around $10 \mathrm{MeV}$, which is a value a little bit larger than those usually calculated in the heavy actinides. In contrast to these nuclei [69-71] the barriers are extremely wide in light actinides. In ${ }^{224} \mathrm{Ra}$ the potential energy oscillates around $10 \mathrm{MeV}$ with increasing elongation and we have not been able to find a second turning point even for very large $Q_{2}$ values. The fission barrier of ${ }^{238} \mathrm{Pu}$ finishes beyond $Q_{2}=100 \mathrm{~b}$. A very extended barrier causes long fission half-lives in all considered nuclei. Also the experimental branching ratio of spontaneous fission to $\alpha$ decay is very small in all of them [27] and they are stable against fission.

In Fig. 1 one can also see a second valley in the PES that goes from the ground state through the reflection-asymmetric shapes with nonzero octupole moment. The huge octupole moment values obtained for small elongation suggests a 


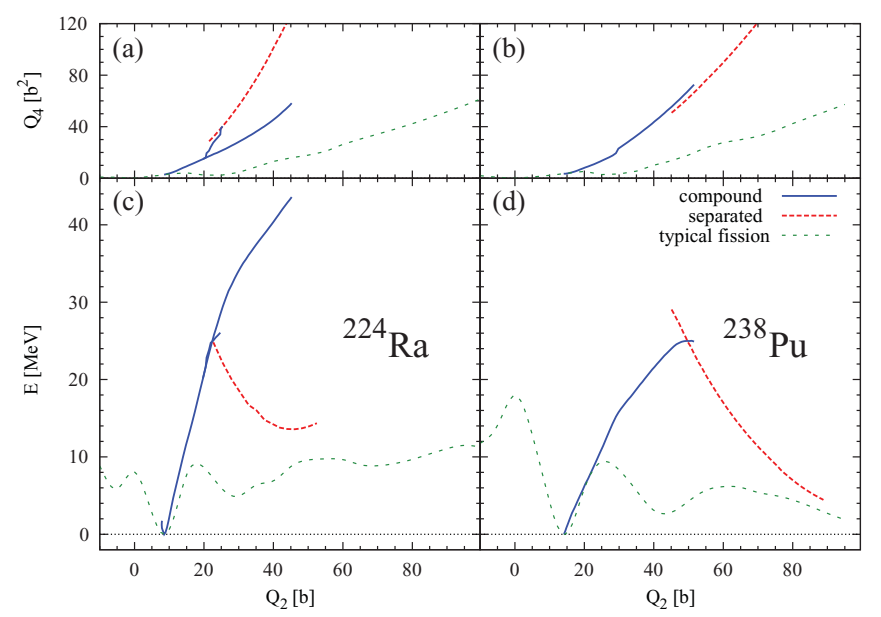

FIG. 2. (Color online) Fission barriers in ${ }^{224} \mathrm{Ra}$ (left) and ${ }^{238} \mathrm{Pu}$ (right) plotted as a function of the quadrupole moment $Q_{2}$ (lower panels). The values of the hexadecapole moment $Q_{4}$ of the nuclei along the fission paths are plotted as a function of quadrupole moment $Q_{2}$ in the upper panels.

large asymmetry in the mass distribution. As the saddle point is reached, the matter density distribution starts to resemble a molecular shape with a small sphere touching a larger one. The large spherical fragment has numbers of protons and neutrons that are consistent with those of ${ }^{208} \mathrm{~Pb}$. This observation points toward a clear relationship between this valley and the phenomenon of CR. We will refer to this valley as "hyper-asymmetric" or as the "CR valley." Along the fission path at the bottom of this valley the elongation of the nucleus rises along with reflection asymmetry. Moreover, the fission path creates a straight line in Fig. 1, as the growth of the quadrupole moment is proportional to the increase of the octupole moment.

In Fig. 2 the hyper-asymmetric fission path is also plotted with a solid blue line as a function of the quadrupole moment. From this figure, it is clear that the hyper-asymmetric barrier is much higher than the classical one. The potential energy grows very quickly with deformation in the CR path up to around $25 \mathrm{MeV}$. Its height is extremely large in comparison with the classical fission barrier. This implies very long half-lives for the decay along this channel (over $10^{10} \mathrm{~s}$ ) and explains why the $\mathrm{CR}$ path was ignored so far as the possible fission path. The experimental evidence of $\mathrm{CR}$, which is characterized by halflives of the same order of magnitude, forces us to consider the hyper-asymmetric path as the possible exotic decay channel.

The evolution of the shapes of nuclei along the CR path from ground state to the saddle point is shown in Figs. 3(a)3(e) for ${ }^{224} \mathrm{Ra}$ and in Figs. 4(a)-4(f) for ${ }^{238} \mathrm{Pu}$. One can see that a cluster of nucleons is budding from the parent nucleus as elongation and asymmetry grow and already at a modest octupole deformation of $Q_{3}=20-30 \mathrm{~b}^{3 / 2}$ a neck starts to be clearly visible in both cases.

Around $Q_{2}=20 \mathrm{~b}$ a bifurcation can be found in the $\mathrm{CR}$ path of ${ }^{224} \mathrm{Ra}$. One of the branches goes toward large deformation parameters $\left(Q_{2}=45 \mathrm{~b}, Q_{3}=55 \mathrm{~b}^{3 / 2}\right)$ with the energy reaching values over $40 \mathrm{MeV}$ above the ground state. This path cannot lead to fission, as the nucleus takes on a conelike shape [see Fig. 3(d)] without a well-defined neck. The

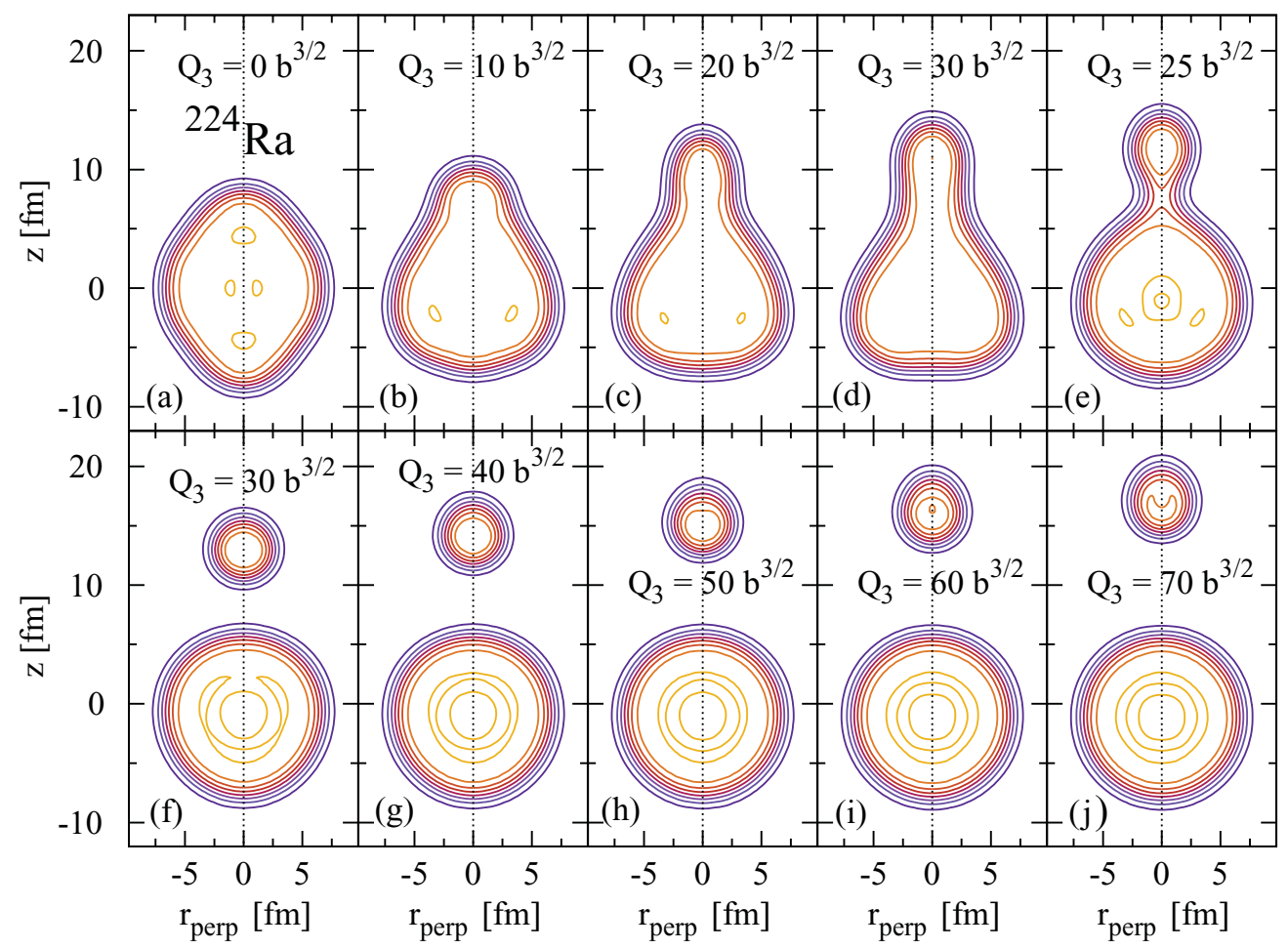

FIG. 3. (Color online) Shape evolution of ${ }^{224} \mathrm{Ra}$ with increasing octupole moment $Q_{3}$. Panels (a)-(d) correspond to the up-going part of the fission path, panel (e) corresponds to the short branch around the saddle point, and panels (f)-(j) correspond to the decreasing part of the fission path. 


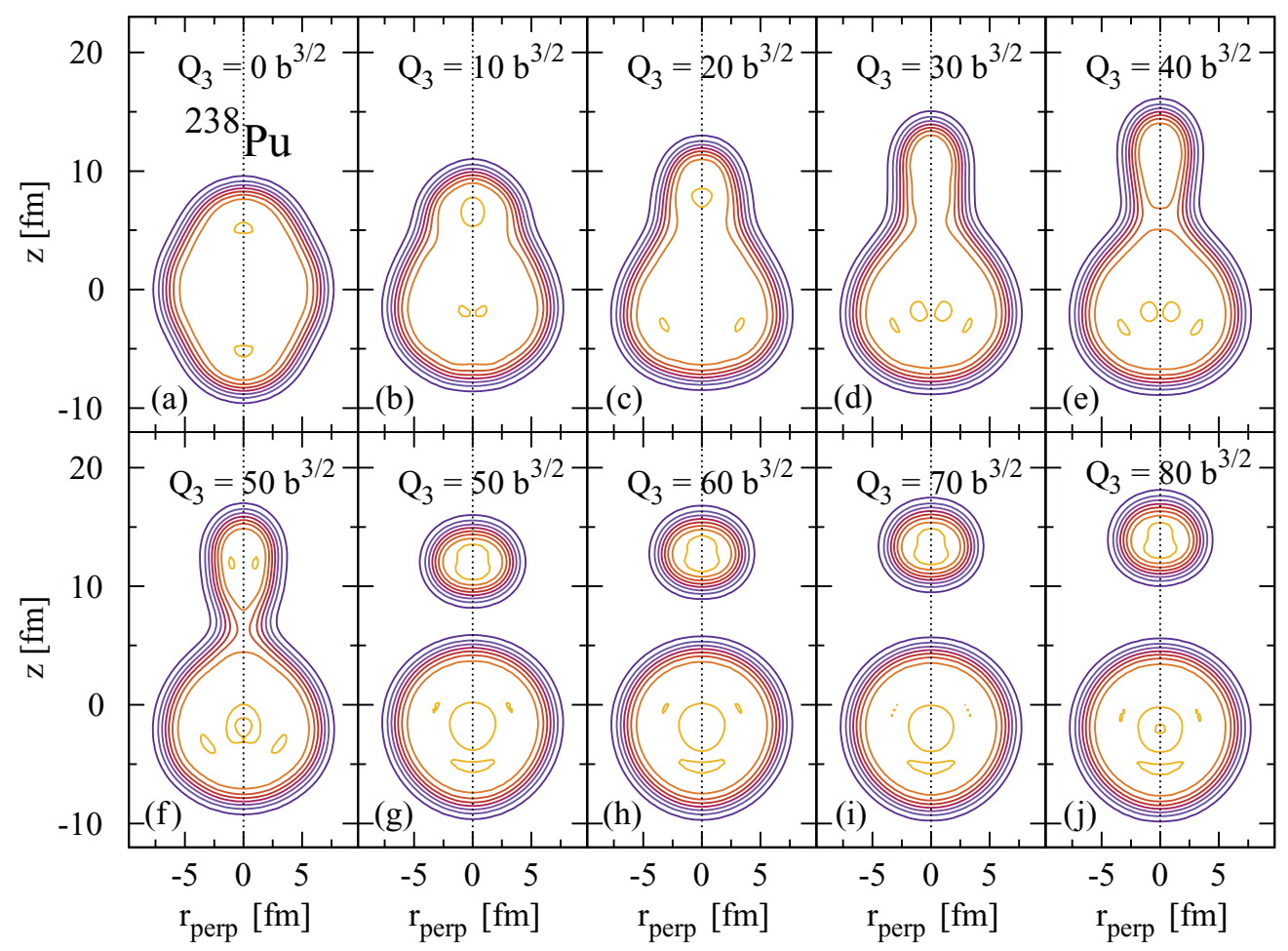

FIG. 4. (Color online) The same as in Fig. 3 but for ${ }^{238} \mathrm{Pu}$. Panels (a)-(f) correspond to the up-going part of the fission path, and panels (g)-(j) correspond to the decreasing part of the fission path.

density profile corresponding to the shorter branch, presented in Fig. 3(e), shows two nearly spherical fragments separated by a neck. This configuration is characterized by a hexadecapole moment which is substantially larger, as seen in Fig. 2(a). The same fission path bifurcation can be found also in ${ }^{222} \mathrm{Ra}$. In a subsequent analysis we will consider only the second, shorter branch as the only relevant one for the description of CR.

In the upper right corners of both panels in Fig. 1 distinct region of the PES is found, with the energy decreasing with increasing deformation. In this part of the PES the system of nucleons is split into two fragments. It may be called: "fusion" valley in contrast to the first part which is commonly called "fission" valley. The "fusion" and "fission" valleys are strongly correlated with each other as they are linked in the same region of the deformation space and the mass splitting between fragments is similar in both cases. The minimum of the energy in this valley creates the descending branch of the CR fission barrier, which is plotted with a red dashed line in Fig. 2. In the upper panels of Fig. 2 we observe the coincidence of the hexadecapole moments of both branches of the barrier at the saddle point.

The density distributions in the "fusion" path are given in Figs. 3(f)-3(j) for ${ }^{224} \mathrm{Ra}$ and in Figs. 4(g)-4(j) for ${ }^{238} \mathrm{Pu}$. Some important information can be deduced from these plots. First, the system is built from two almost spherical fragments. The space between their surfaces is wide for at least a few femtometers and increases with $Q_{2}$ and $Q_{3}$. The heavier fragment is the doubly-magic spherical ${ }^{208} \mathrm{~Pb}$ after scission of ${ }^{224} \mathrm{Ra}$ or ${ }^{210} \mathrm{~Pb}$ in the case of ${ }^{238} \mathrm{Pu}$. [Those values are in good agreement with the mass and charge of the heavy pre-fragments of configurations (e) in ${ }^{224} \mathrm{Ra}$ and (f) in ${ }^{238} \mathrm{Pu}$ before scission; cf. also Sec. IIIC.] The lighter fragment may be slightly deformed (prolate or oblate). Its shape is mostly determined by the shape of the ground state of the corresponding nucleus as the Coulomb interaction with the heavier fragment is not strong enough. In the case of ${ }^{30} \mathrm{Mg}$ emitted from ${ }^{238} \mathrm{Pu}$, the ground state is oblate $\left(\beta_{2}=-0.222\right)$ [98]. In the other nucleus, the spherical ${ }^{14} \mathrm{C}$ isotope constitutes the lighter fragment of the CR from ${ }^{224} \mathrm{Ra}$.

Once the system has split in two, the shapes of the fragments do not change significantly as they move apart and the increase of the total quadrupole and octupole momenta is a consequence of the increasing distance between the fragments. Therefore the change in the potential energy after scission is mainly due to the decreasing Coulomb repulsion and it should decline hyperbolically with the distance between the centers of fragments, which is roughly proportional to $Q_{2}$. Such behavior can be seen in Fig. 2 close to the saddle point. However, for larger deformations we observe a departure from the expected behavior that calls for a larger basis. Unfortunately, the use of a larger basis can be problematic as a consequence of numerical instabilities in the evaluation of matrix elements due to finite computer accuracy. Those instabilities lead in some cases to strange behaviors in the energy, preventing the use of a very large basis (see also Appendix A where the convergence of the energy is discussed). To avoid these difficulties, which are critical for the determination of half-lives in the WKB scheme, we have adopted an approximate strategy to be discussed in Sec. III B below. The insufficient size of the basis also manifests in the matter distributions of the lighter fragment seen in panels (i) and (j) of Fig. 3, where an unnatural stretching toward large $z$ values can be noticed. 
The solution of the HFB equation often depends on the nuclear matter distributions of the initial wave function used in the iterative procedure. In many regions of the PES, especially close to the scission line, two solutions may be obtained for the same constraints. If the calculation begins with a compact shape of the nucleus, the final solution will have similar properties. If a configuration with two separated fragments is chosen as the initial condition, again a solution with similar properties will be found. When the same constraints are put on the system the two results will have the same quadrupole and octupole momenta but they may have different higher multipolarities as well as energy. Because of this, the "fusion" valley extends toward ground state much further than is shown in Fig. 1. It covers the area around the "fusion" path in the $Q_{2}-Q_{3}$ deformation space. Its part is hidden below the "fission" valley shown in Fig. 1. In this figure we have marked both the fission paths and "fission" valley but not the whole "fusion" valley.

\section{B. Tracking fission paths as a function of octupole moment}

Tracking the hyper-asymmetric fission path in the PES is a difficult task from a numerical standpoint. Usually, the fission path is determined by searching for the local minima of the energy along cuts of constant $Q_{2}$. This method could also be applied to the CR path as is shown in Fig. 5, where the potential energies of the ${ }^{238} \mathrm{Pu}$ nucleus are plotted as a function of $Q_{3}$ for fixed values of $Q_{2}$. It is clear that a local minimum corresponding to the hyper-asymmetric fission can be determined in most of the cases, usually at higher energies than the minimum of the classical fission observed at $Q_{3}=0$ $\mathrm{b}^{3 / 2}$. However, in many nuclei there are certain $Q_{2}$ values where a plateau is observed instead of a well-defined minimum [e.g., for $Q_{2}=30 \mathrm{~b}$ in ${ }^{238} \mathrm{Pu}$ in Fig. 5(d)]. This problem can be solved by using an alternative choice of coordinate to describe the formation of the daughter nuclei. As mentioned before, in the CR path $Q_{2}$ is roughly proportional to $Q_{3}$ and therefore the octupole moment can also be used as the leading coordinate.

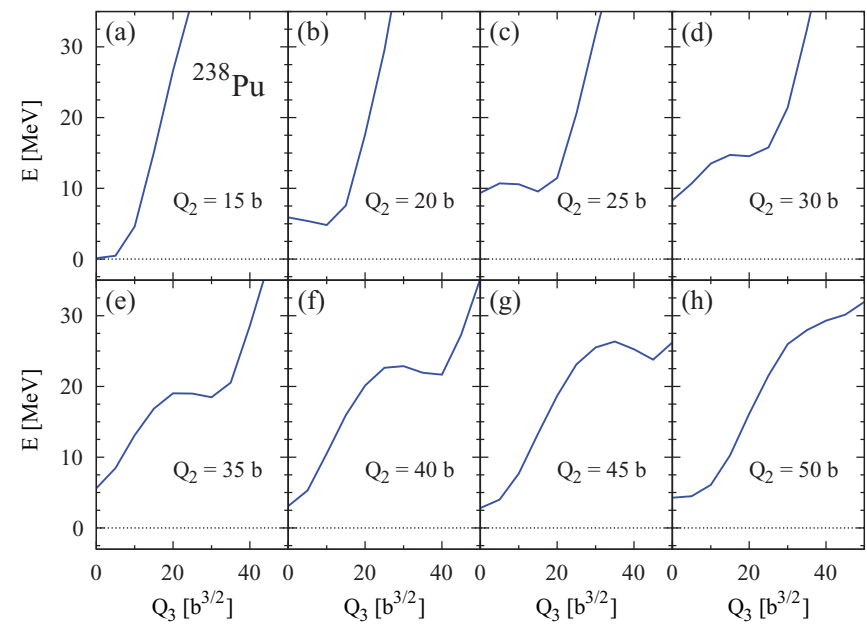

FIG. 5. (Color online) Potential energies of ${ }^{238} \mathrm{Pu}$ as a function of the octupole moment $Q_{3}$ for several values (shown in each panel) of the quadrupole moment $Q_{2}$.

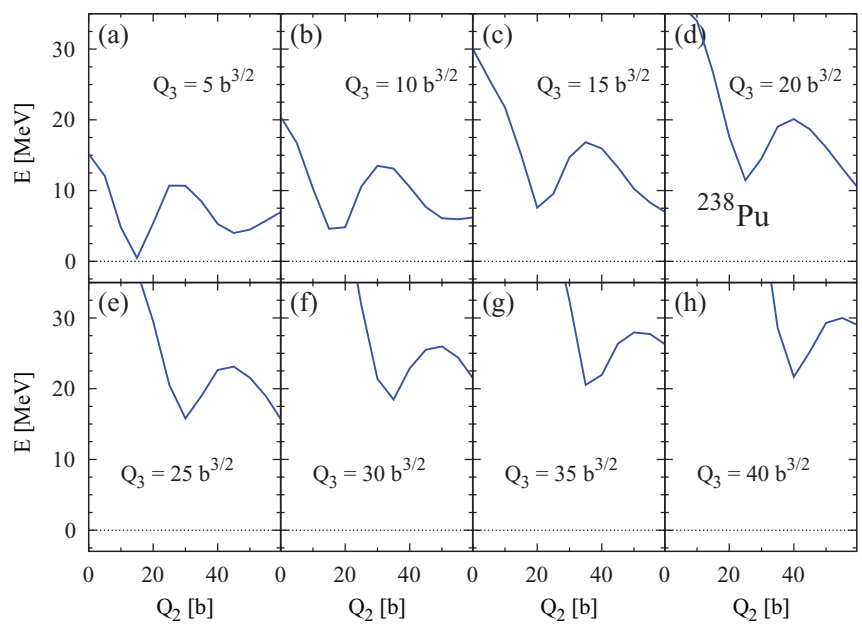

FIG. 6. (Color online) Potential energies of ${ }^{238} \mathrm{Pu}$ as a function of the quadrupole moment $Q_{2}$ for several values (shown in each panel) of the octupole moment $Q_{3}$.

The potential energy for fixed $Q_{3}$ as a function of $Q_{2}$ is plotted in Fig. 6. Here the hyper-asymmetric valley is clearly visible at every point and it is trivial to determine local minima there and track the fission path. The octupole moment can also be used as the driving coordinate to determine half-lives in the WKB approximation, as already described in Sec. II. We conclude that the octupole moment is better suited than the quadrupole moment to describe the CR paths in the PES and therefore we will use it as a leading coordinate in the following discussion.

The profiles of the CR path in ${ }^{224} \mathrm{Ra}$ and ${ }^{238} \mathrm{Pu}$, presented already as a a function of quadrupole moment in Fig. 2, are plotted now as a function of octupole moment in Figs. 7(e) and 7(f). Initially, the energy increases with increasing octupole

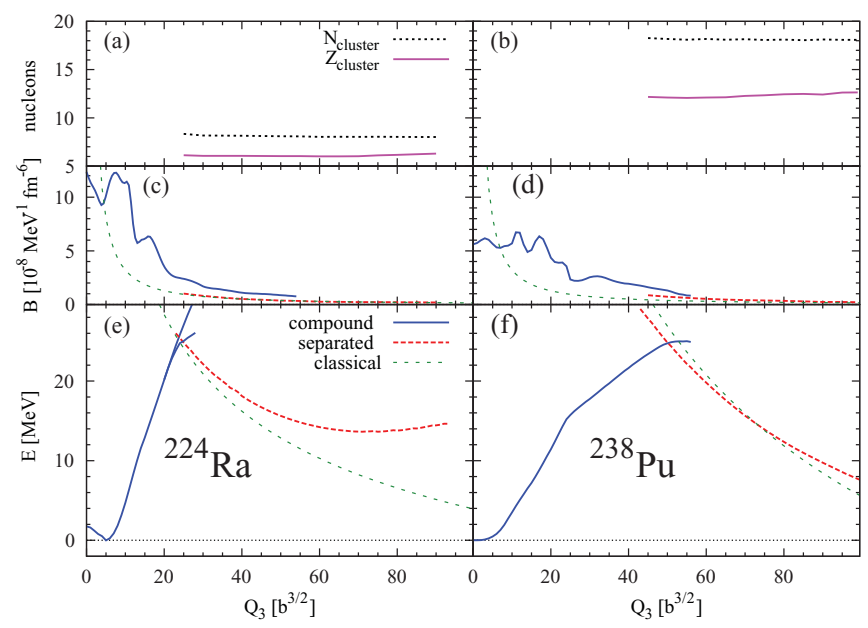

FIG. 7. (Color online) Hyper-asymmetric fission barriers in ${ }^{224} \mathrm{Ra}$ (left) and ${ }^{238} \mathrm{Pu}$ (right) as a function of the octupole moment $Q_{3}$ (lower panels). Approximate Coulomb repulsion energies [Eq. (8)] for corresponding clusters are also plotted. In the middle panels, the mass parameter $B\left(Q_{3}\right)$ calculated in a microscopic way is plotted. In addition, the classical value [Eq. (11)] corresponding to two separate fragments is also given. In the upper panel, the number of nucleons in clusters is given as a function of the octupole moment $Q_{3}$. 
moment in an almost quadratic fashion from the ground state, which may be refection symmetric $\left({ }^{238} \mathrm{Pu}\right)$ or asymmetric $\left({ }^{224} \mathrm{Ra}\right)$. The slope of energy decreases when approaching the top of the barrier. At some point the branch with two fragments becomes the lowest energy solution with an energy which is essentially the Coulomb repulsion of the fragments expressed as a function of the octupole moment of the two fragments. The Coulomb repulsion energy can be very well be approximated by the classical value corresponding to two uniformly charged spheres:

$$
V\left(Q_{3}\right)=V_{\text {Coul }}-Q=e^{2} \frac{Z_{1} Z_{2}}{R}-Q,
$$

where $R$ represents the distance between the centers of mass of the fragments. Asymptotically, the total energy tends to the $Q$ value of the reaction that can be extracted from the experimental binding energies [97]. The connection between the variable $R$ and the octupole moment $Q_{3}$ is obtained in a simple geometrical way when the two fragments are spherical or when two point masses are considered:

$$
Q_{3}=f_{3} R^{3},
$$

where

$$
f_{3}=\frac{A_{1} A_{2}}{A} \frac{\left(A_{1}-A_{2}\right)}{A}
$$

is given in terms of the total mass number $A$, and the mass numbers of each of the fragments, $A_{1}$ and $A_{2}$. In Figs. 7(e) and 7(f) we observe that around the saddle point both the HFB and the approximate Coulomb repulsion energy of Eq. (8) coincide with a noticeable agreement of the order of $2 \mathrm{MeV}$. Small differences can be mainly attributed to the excitation of the lighter fragment in the presence of the Coulomb field of the heavy-mass residue as well as to the deformation of the emitted cluster that can be different from the one of its ground state. As already mentioned in Sec. III A, at larger $Q_{3}$ values the HFB energy results are more affected by the finite size of the basis used and therefore they lie at an energy higher than the one of an infinite basis calculation. Because this range of octupole moments is very relevant for the determination of half-lives in the WKB framework, we will use the approximate expression of Eq. (8) in the calculation of half-lives instead of the HFB energy.

The collective mass $B\left(Q_{3}\right)$ linked to the octupole moment is also plotted in Figs. 7(c) and 7(d). The collective mass of the compound system computed microscopically substantially differs from the semiclassical value given by the reduced mass of the two fragments, $\mu=m_{n} A_{1} A_{2} /\left(A_{1}+A_{2}\right)$ (a quantity connected to the kinetic energy for the coordinate $R$ ) but written in terms of $Q_{3}$ :

$$
B\left(Q_{3}\right)=\frac{\mu}{9 Q_{3}^{4 / 3} f_{3}^{2 / 3}} .
$$

This quantity derived from the ATDHFB model in Eq. (4) varies considerably when the nucleus is stretched out. This is a consequence of the strong dependence of collective mass on the single-particle effects that show up during the development of the neck. After scission the microscopic collective mass $B\left(Q_{3}\right)$ is very close to the classical value, as expected, and therefore the contribution to the action $[\mathrm{Eq}$ (3)] from the postscission region will be very similar in our calculations to the ones of the analytical superasymmetric fission model (ASAFM) of Ref. [38].

In Figs. 7(a) and 7(b) we have shown the numbers of protons and neutrons of the lighter fragment after scission in the CR path. In this way the cluster emitted in the hyper-asymmetric fission can be identified. In ${ }^{224} \mathrm{Ra}$ it corresponds exactly to the experimentally observed cluster ${ }^{14} \mathrm{C}$. The PES of ${ }^{238} \mathrm{Pu}$ indicates ${ }^{30} \mathrm{Mg}$ as a potential cluster. This is one of the clusters observed in the decay of this nuclide $\left({ }^{28,30} \mathrm{Mg}\right.$ and $\left.{ }^{32} \mathrm{Si}\right)$.

We would like to point out an important aspect of tracking the fission path after the scission point. In the laboratory it is not possible to transfer nucleons between the daughter nuclei once the fragments are created. We have checked that the numbers of neutrons and protons are usually constant in the minimum of the "fusion" valley, although they may differ slightly from integer numbers. Imposing given fragment masses will lead to configurations with higher energies. The "fusion" paths for those systems with mass asymmetry differing by a few nucleons from the one corresponding to the minimum energy configuration run parallel to the minimum energy path and they reach the scission point at almost the same position in the $Q_{2}-Q_{3}$ deformation space, i.e., in the saddle, with similar energy. A tiny instability around the saddle may lead to an alternative choice of cluster configuration. The length of the fission barrier corresponding to each possible nascent fragment would determine which one will be observed in the experiment. Further detailed investigations should be performed using additional constraints on the number of nucleons of each fragment.

\section{Scission point transition from a compound nucleus to two separated fragments}

Two independent branches are clearly visible in the CR fission barriers of Figs. 2 and 7. As described in Sec. III B they differ substantially in the shapes of the nucleus corresponding to each of them. In the first, the up-going part of the barrier, called the "fission" path, the shape corresponds to that of a compound nucleus [Figs. 3(a)-3(e) and 4(a)-4(f)]. For the deformations around the ground state the corresponding shape is not too distant from the ellipsoid and therefore we can say that the nucleus takes a compact shape. However, for large deformations a neck can be clearly distinguished in this branch and the density distribution of the nucleus is of molecular type. A completely different type of shape is obtained on the downslope side of the barrier, called the "fusion" path [Figs. 3(f)3(j) and 4(g)-4(j)]. Two well-separated nuclei can be observed there as the matter density in the region between them goes to zero and the shortest distance between the nuclear surfaces of the two fragments is at least a few femtometers.

In spite of the different shapes of the density profiles along the two branches, they share many similar nuclear properties at the top of the barrier. For instance, comparable values of the quadrupole, octupole, and hexadecapole moments can be found there. The density distribution before scission is close to the one after separation and the only important differences can be found in the neck region. Moreover, the energies also have similar values and we can easily find in Fig. 7 a crossing 
point where the potential energy on the two branches is the same for some value of $Q_{3}$. A first and rough approximation could be to consider this as the scission point. This assumption can be used to get a quite reasonable estimation of the size of the barrier and fission half-lives. Nevertheless, a more precise analysis shows that the shape of the system is clearly different in the two branches and none of them can be considered as two touching fragments.

The passage from a compact shape to a two-fragment one cannot be treated as an instant transition at the crossing point. Some energy barrier, not seen clearly in the PES spanned in the $Q_{2}-Q_{3}$ space, exists between the "fission" and the "fusion" path. These two constraints are not sufficient to describe the continuous path connecting the two branches. In such a path, the nuclear density in the neck would decrease gradually to zero and then the two fragments would be disengaged. The relevant parameter along this path is the neck parameter $[69,74]$ defined through the mean value of the operator

$$
\hat{Q}_{N}=\exp \left(\frac{\left(z-z_{0}\right)^{2}}{a^{2}}\right) \text {. }
$$

The value of the neck parameter roughly corresponds to the number of nucleons in a slice perpendicular to the $z$ axis, centered at the position $z_{0}$ and of width $a$. In the present case we have chosen $a=0.1 \mathrm{fm}$, which gives us a sufficiently thin slice, and $z_{0}=7.5 \mathrm{fm}$, which corresponds to the position of the neck. The neck parameter is correlated with the hexadecapole moment, a quantity that has been used routinely in fission calculations [74] to study the scission process, but the neck parameter is more suited to drive the system through scission when $z_{0}$ and $a$ are chosen conveniently. The quantity $Q_{N}$ never goes to zero in any physical situation because of the nonvanishing tail of the nuclear density distribution but it can be arbitrarily small if the slice is properly located in the region between the two separated fragments.

In Figs. 8(a) and 9(a) the PES of ${ }^{224} \mathrm{Ra}$ and ${ }^{238} \mathrm{Pu}$ are plotted, respectively, as a function of the octupole moment $Q_{3}$ and the neck parameter $Q_{N}$. In these plots, we only show the relevant region around the top of the barriers. The minima of the valleys on this surface are marked by green dashed lines. The "fission" path goes from $Q_{3}=20 \mathrm{~b}^{3 / 2}, Q_{N}=0.65$ to $Q_{3}=28 \mathrm{~b}^{3 / 2}, Q_{N}=0.18$ in ${ }^{224} \mathrm{Ra}$ and from $Q_{3}=45 \mathrm{~b}^{3 / 2}$, $Q_{N}=0.45$ to $Q_{3}=55 \mathrm{~b}^{3 / 2}, Q_{N}=0.20$ in ${ }^{238} \mathrm{Pu}$. The "fusion" path is marked by an almost horizontal line with neck parameter in the range from $Q_{N}=0.02$ to $Q_{N}=0.05$ in both nuclei. In Fig. 8(a) a horizontal line at $Q_{N}=0.65$ is

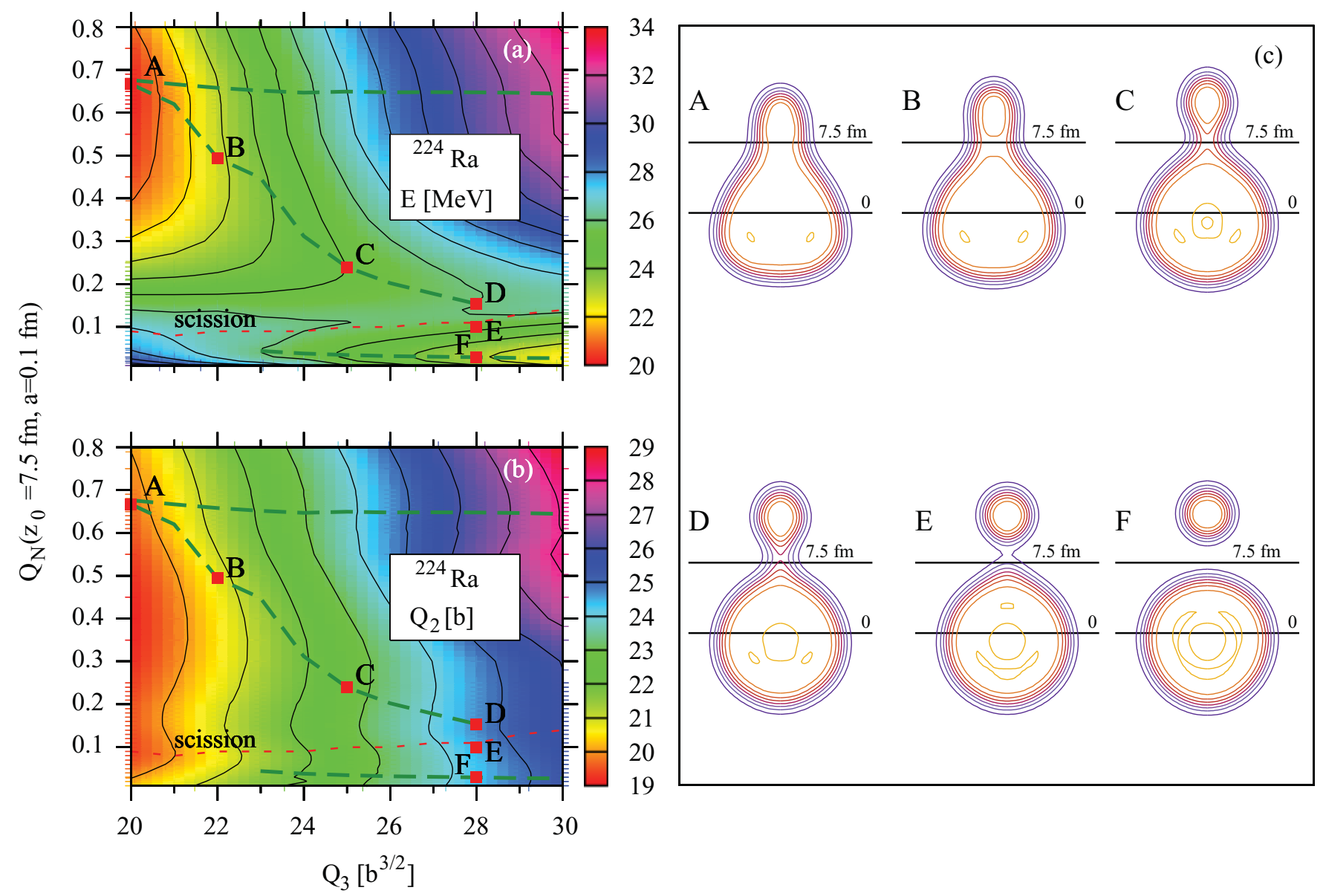

FIG. 8. (Color online) (a) Saddle region of the PES and (b) quadrupole moment $Q_{2}$ plotted as a function of octupole moment $Q_{3}$ and neck parameter $Q_{N}$ in ${ }^{224} \mathrm{Ra}$. Green dashed lines are fission paths and the red short-dashed line is the scission line. (c) Contour plots of density distributions for some deformations marked in panel (a). Equidensity lines are plotted every $0.02 \mathrm{fm}^{-3}$. 

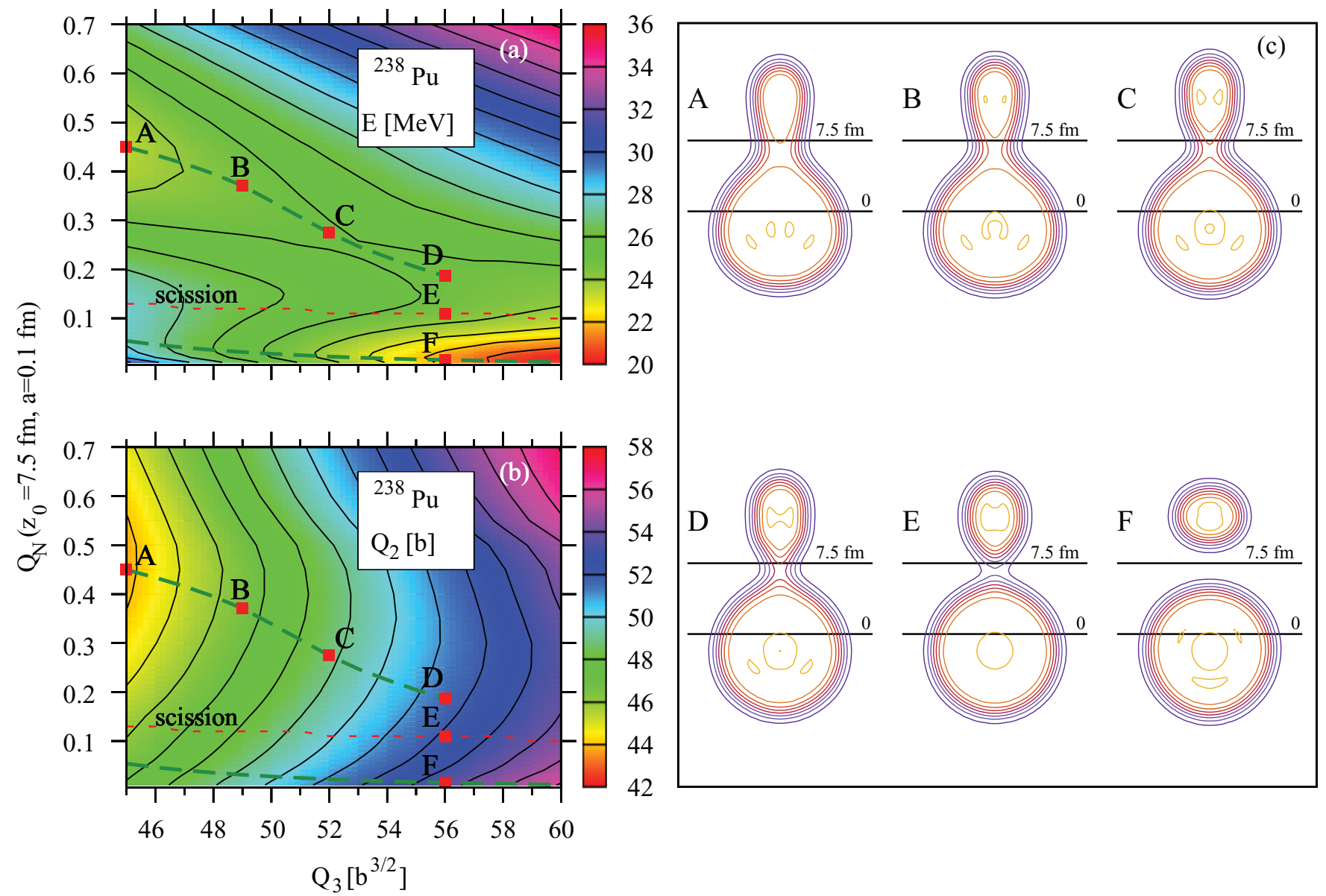

FIG. 9. (Color online) The same as in Fig. 8 but for the ${ }^{238} \mathrm{Pu}$ nucleus.

also shown. It corresponds to the branch of the fission path in ${ }^{224} \mathrm{Ra}$ that goes up in energy and that shows shapes that do not develop a sizable neck. The red short-dashed line around $Q_{N}=0.10$ is the scission line describing these configurations where the density in the neck region goes below $0.4 \mathrm{fm}^{-3}$. It lies along the ridge on the energy surface separating "fission" and "fusion" valleys. Owing to the use of the neck parameter both valleys are linked in a continuous way along the whole scission line and there is no sudden energy change.

In Figs. 8(b) and 9(b) the quadrupole moments of the ${ }^{224} \mathrm{Ra}$ and ${ }^{238} \mathrm{Pu}$ are plotted in the same space of deformations as in Figs. 8(a) and 9(a). We observe how the quadrupole moment increases monotonically with increasing octupole moment. The variations with the neck parameter are much smaller though. This explains why the quadrupole moment does not provide a quantity sensitive enough for the detailed description of the rupture of the nucleus into two pieces. The hexadecapole moment is also insensitive to changes of the neck parameter and it varies by not more than $4 \mathrm{~b}^{2}$ for the fixed octupole moment in the configurations considered in the figures. A larger monotonic increase of $Q_{4}$ with $Q_{3}$ can be observed.

Finally, in Figs. 8(c) and 9(c) the matter density distribution at the different stages of the scission process [marked by the letters A, B, C, D, E, and F in panels (a) and (b)] is shown. Following the points at the "fission" path marked as A, B, C, and $\mathrm{D}$ a reduction of the neck parameter can be noticed. The neck becomes thinner and a decrease of the nuclear density up to half of the bulk value in configuration $\mathrm{D}$ can be observed. Between configurations D, E, and F the scission process takes place and the shape of the lighter fragment evolves from prolate in D to spherical or oblate in F. It is also interesting to notice that shapes D, E, and F have essentially the same octupole moment and a very similar quadrupole moment.

We also observe in Figs. 8(a) and 9(a) that the crossing point of the two branches ("fission" and "fusion") at $Q_{3}=50 \mathrm{~b}^{3 / 2}$ for ${ }^{238} \mathrm{Pu}$ and at $Q_{3}=25 \mathrm{~b}^{3 / 2}$ for ${ }^{224} \mathrm{Ra}$ are well separated as they correspond to different values of $Q_{N}$. It is now clear that to pass directly from one configuration to the other it is necessary to climb the "neck barrier," which is over $1 \mathrm{MeV}$ high, although the energy of both the "fission" and "fusion" paths is the same. From these plots it becomes clear that it is energetically preferable to follow the "fission" path to the very end, where the neck is very thin (see the shape of the nucleus at point D) and there is no barrier separating the nucleus from the scission line, than to climb the "neck barrier." The subsequent evolution of the shape of the nucleus should follow the direction corresponding to the maximal decrease in energy (the gradient direction). In fact, this means that the neck parameter should decrease rapidly almost without change of the octupole moment until it reaches the bottom 
of the "fusion" valley. In this way the nucleus takes first the shape corresponding to configuration $\mathrm{E}$ and then the one corresponding to configuration F at the "fusion" path. At this point, the direction of largest energy slope corresponds to the "fusion" path. The nuclear profile E really represents a scission configuration with two nuclei that have only a common tail of the density distribution below the value of $0.04 \mathrm{fm}^{-3}$.

In this way we can follow step by step the sequence of changes of the nuclear shape around the scission point without losing continuity. From the present analysis we can obtain a precise outline of the CR fission barrier in the actinides. The first fragment of the barrier is built from the whole "fission" branch where energy increases with deformation. After its termination a rapid decrease of the energy takes place without changes in quadrupole or octupole moment until the "fusion" path is reached. Finally, a further decrease of energy with increasing deformation is observed in the "fusion" path. Although such a shape seems to lose continuity in the plot of the energy barrier as a function of quadrupole or octupole moment (see Figs. 2 and 7), it is continuous in the space spanned by the neck parameter.

Let us now take a closer look to the shapes of the nucleus at the end of the "fission" path and just after reaching the "fusion" path. i.e., from points D and F in Figs. 8 and 9. These configurations differ in energy by a few $\mathrm{MeV}$ and they are distinguished by their matter distribution at the neck. Nevertheless, the distribution of nuclear matter is quite similar in the two cases. In Fig. 10(a) the density distributions of the nucleus ${ }^{224} \mathrm{Ra}$ before scission (on the left-hand side) and after scission (on the right-hand side) are shown. Both nuclear systems have the same octupole moment $Q_{3}=28 \mathrm{~b}^{3 / 2}$ and similar quadrupole and hexadecapole moments ( $Q_{2}=24.7 \mathrm{~b}, Q_{4}=33.8 \mathrm{~b}^{2}$ for the compound shape and $Q_{2}=24.9 \mathrm{~b}, Q_{4}=36.6 \mathrm{~b}^{2}$ after scission). First, we notice
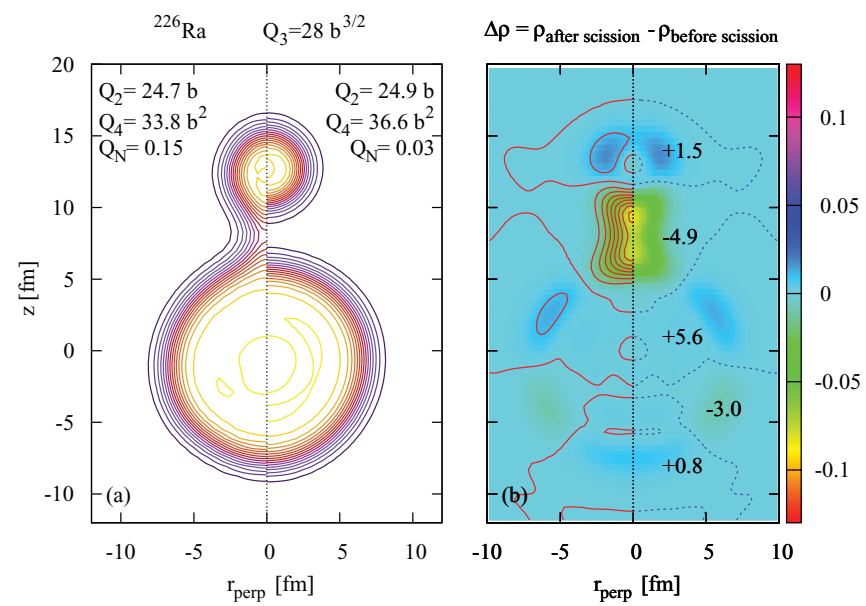

FIG. 10. (Color online) (a) A comparison of the density distribution of ${ }^{224} \mathrm{Ra}$ before and after scission at $Q_{2}=28 \mathrm{~b}^{3 / 2}$. Equidensity lines are plotted every $0.01 \mathrm{fm}^{-3}$. (b) A plot of the differences between the two matter density distributions of panel (a). In this plot, contour lines in the left part are plotted every $0.01 \mathrm{fm}^{-3}$, whereas on the right-hand side only for $\Delta \rho=0$. The number of nucleons shifted to $(+)$ or from $(-)$ the marked region are also given.
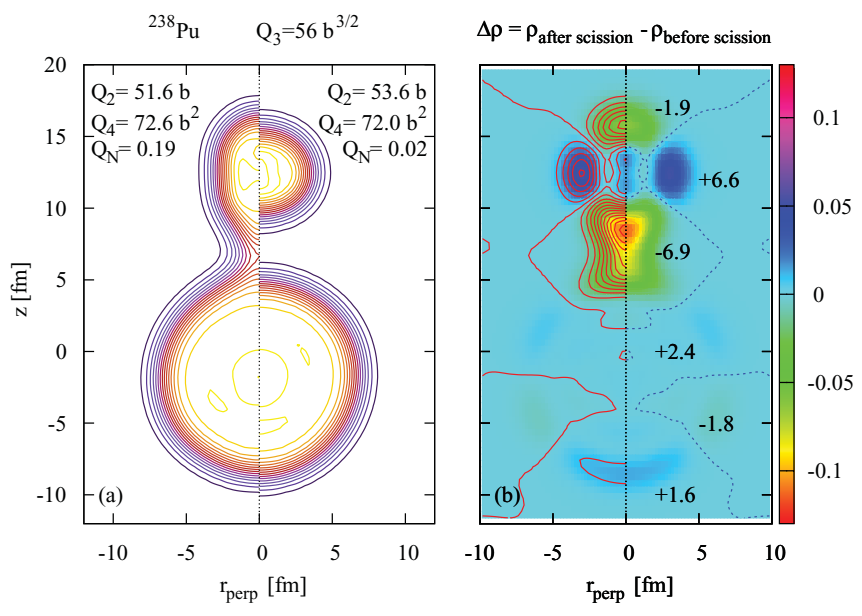

FIG. 11. (Color online) The same as in Fig. 10 but for the ${ }^{238} \mathrm{Pu}$ nucleus at $Q_{3}=56 \mathrm{~b}^{3 / 2}$.

that in both cases the larger fragment is the same spherical doubly-magic ${ }^{208} \mathrm{~Pb}$ nucleus. It is well developed before the rupture of the neck and only a small transfer of nuclear matter can be seen within this part of the system. The smaller fragment ${ }^{14} \mathrm{C}$ is also present before scission. Its central part is well separated from the heavier fragment and its spherical shape is almost developed before rupture of the neck, although a small prolate deformation can be noticed. The nuclear density in the neck is lower than the bulk nuclear density and goes down to $0.09 \mathrm{fm}^{-3}$ in the molecular configuration. The distance between the centers of the two incipient fragments before scission is the same as that between the separated fragments after scission.

For a further analysis of the matter distribution at the scission point configuration let us look at Fig. 10(b), where the differences between the density distributions depicted in Fig. 10(a) are shown. Only approximately 4.9 nucleons are transferred from the neck to the fragments. Small shifts of nuclear matter can also be observed within each of the fragments. The heavy fragment is not changing in a remarkable way. The light fragment is relatively more affected by the displacement of nuclear matter and changes of its shape.

A similar conclusion can be deduced for ${ }^{238} \mathrm{Pu}$ from Fig. 11. On both sides of Fig. 11(a) the nucleus has the same octupole moment $Q_{3}=56 \mathrm{~b}^{3 / 2}$ and similar quadrupole and hexadecapole moments $\left(Q_{2}=51.6 \mathrm{~b}, Q_{4}=72.6 \mathrm{~b}^{2}\right.$ for the molecular shape and $Q_{2}=53.6 \mathrm{~b}, Q_{4}=72.0 \mathrm{~b}^{2}$ for two fragments). Changes in the density distribution in the cluster region are slightly larger than in ${ }^{224} \mathrm{Ra}$, since a change of deformation in the lighter fragment from prolate to oblate can be noticed. The ${ }^{30} \mathrm{Mg}$ nucleus corresponding to the lighter fragment has a ground state which is very soft against changes of its quadrupole deformation [99]. Before scission the lighter fragment of the compound nucleus is stretched to have a prolate shape whereas after scission it takes its oblate ground-state deformation ( $\beta_{2}=-0.222$ [98]).

The constraint on the neck parameter allows for a detailed analysis of the scission point configuration. As a consequence 
a continuous fission path going from the compound nucleus to the two separated fragments can be determined. The precise localization of the saddle point can also be settled using this parameter.

\section{RESULT FOR ACTINIDE NUCLEI}

The previous section contains a complete analysis of two relevant examples of cluster decay in the actinides. Despite the differences in masses of the two isotopes considered there, it is clear that the same mechanism is responsible for CR in all the nuclei in this region. In this section, the results for the other isotopes are presented. We concentrate on the CR fission barriers and on the half-lives for this very mass asymmetric decay. A thorough comparison of our theoretical results with experimental data will be also discussed in this section.

In Fig. 12 the fission barriers are presented as a function of the octupole moment $Q_{3}$ for all actinide nuclei in which

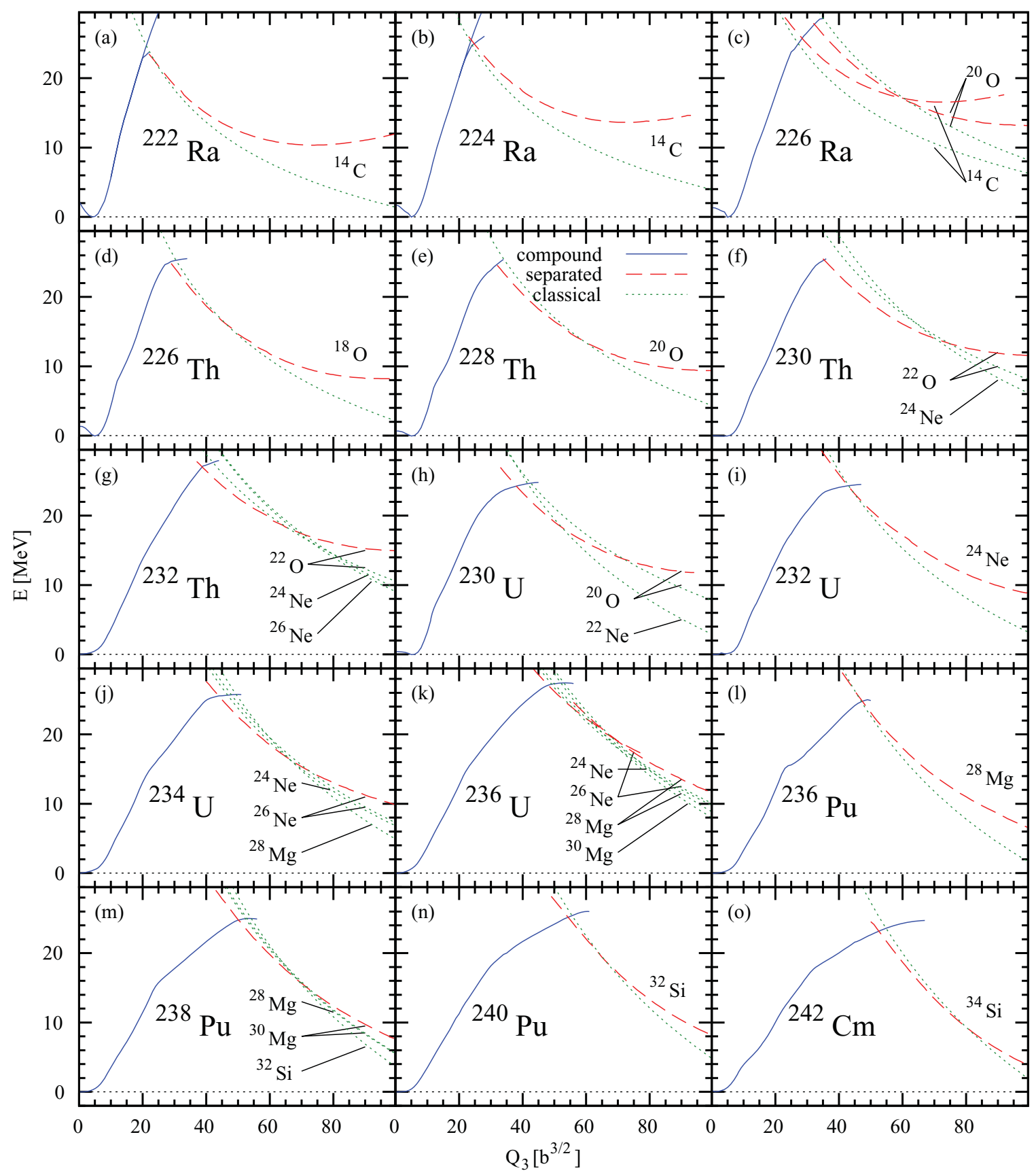

FIG. 12. (Color online) Hyper-asymmetric fission barriers for all considered isotopes as a function of octupole moment $Q_{3}$. The fragment of the barrier with the compound nucleus is marked with a blue solid line and that with two separated fragments with a red dashed line. Green dotted lines show classical Coulomb energy for two fragments. The corresponding clusters are indicated for solutions after scission. 
cluster emission was experimentally investigated. The height of the barrier remains roughly constant for all the considered nuclei and it only varies between 22 and $26 \mathrm{MeV}$. The saddle point is located at small octupole deformations $Q_{3}=20 \mathrm{~b}^{3 / 2}$ for light nuclei and it gradually shifts to $Q_{3}=55 \mathrm{~b}^{3 / 2}$ for the heaviest ones.

In some nuclei we have plotted curves for two or three possible mass distributions of the clusters obtained with Eq. (8). We do so also when the light fragment corresponding to the minimum of the $\mathrm{CR}$ valley does not agree with the mass of the cluster observed experimentally. In these cases we consider both clusters measured experimentally and predicted by the model as possible decay products and we determine the corresponding CR barriers and spontaneous emission half-lives for all possible clusters. In some nuclei the mass of the emitted cluster has not been precisely identified by the experiment (e.g., for ${ }^{236} \mathrm{U}$ ). In these isotopes we have calculated barriers for all possible clusters as well. It is worth recalling that all the curves converge close to the saddle point. This is because at the saddle point a difference of two neutrons or protons in any of the fragments does not change substantially the shape of the density distribution or energy of the whole system. Therefore, the nucleus at the scission point can chose any of the decay channels.

The flat shape of the hyperbolic curves of Eq. (8) at high deformation leads to important differences in the length of the barrier for each decay product. This is the reason for the few orders of magnitude differences in the half-lives of each of the cluster emission reactions.
Half-lives calculated for all considered cluster emissions are plotted in Fig. 13. Corresponding data are also presented in Table I. The theoretical results are compared with known experimental data where available. As can be seen in this figure, the measured half-lives are reproduced with an accuracy of two or three orders of magnitude in most of the cases. This is the typical kind of agreement observed in all the other models of cluster emission.

In Fig. 13 we have presented half-lives calculated with the energy of the "fusion" path given in Eq. (8). This classical expression does not include the pure quantal effect of the ZPE. Therefore we have shown also the results with the ZPE calculated in the microscopic way subtracted from the Coulomb energy. Table I contains only half-lives calculated in the latter manner.

In ${ }^{226} \mathrm{Ra}$ we have found two parallel fission paths after the saddle point. Such a configuration of the PES suggests two modes of hyper-asymmetric fission with two different clusters that can be produced. The results of half-lives on both paths show much faster decay with emission of experimentally observed ${ }^{14} \mathrm{C}$ than with ${ }^{20} \mathrm{O}$ determined in the other "fusion" path.

In a few cases $\left({ }^{226} \mathrm{Ra},{ }^{230-232} \mathrm{Th}\right.$, and $\left.{ }^{230} \mathrm{U}\right)$ the clusters predicted by the HFB calculations are not observed experimentally. A possible explanation is that the half-lives of the experimentally observed decays are shorter by a few orders of magnitude than the unobserved decays predicted by our calculations. This can be seen, for instance, for the ${ }^{230} \mathrm{Th}$ decay in Fig. 12, where the energy curve for the experimentally

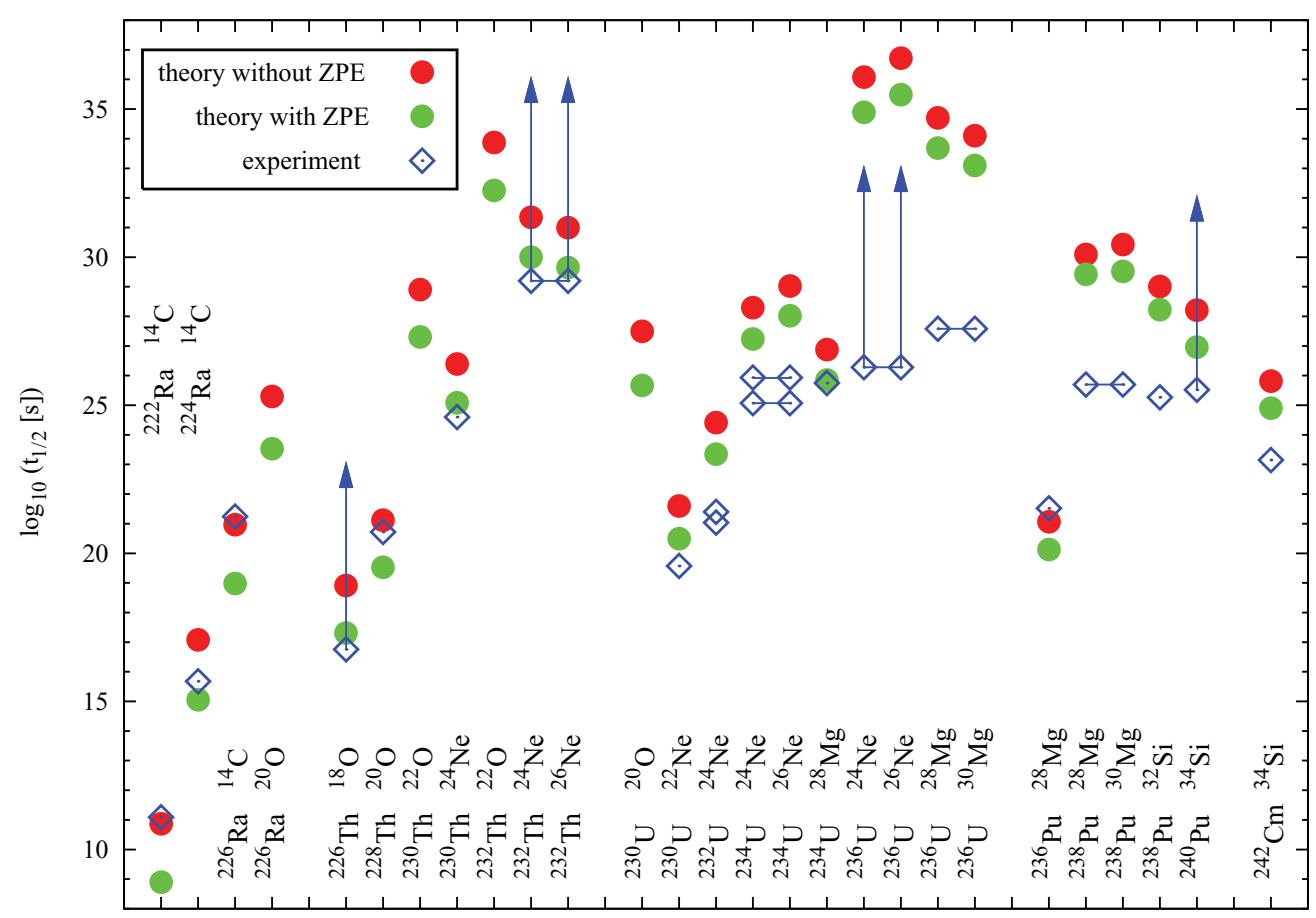

FIG. 13. (Color online) Half-lives for cluster emission of various isotopes and various clusters. Blue diamonds show the experimental half-lives. Arrows indicate the low experimental limit. Connected diamonds are for experimental values for two clusters. If experimental data from different experiments differ by more than 0.3 , the extreme values are indicated. 
TABLE I. Half-lives for cluster emission from the light actinides calculated in the HFB mean-field approximation compared with the experimental data where available. Experimental data in each section correspond to one or two emitted nuclei as indicated. The theoretical results include the ZPE correction; for the corresponding values without it, see Fig. 13. In general terms the half-lives without ZPE are one to two orders of magnitude larger than the ones computed with the ZPE.

\begin{tabular}{lccccc}
\hline \hline Emitter & Cluster & Residue & $\log \left(t_{1 / 2}^{\mathrm{HFB}}[\mathrm{s}]\right)$ & $\log \left(t_{1 / 2}^{\exp }[\mathrm{s}]\right)$ & Ref. \\
\hline${ }^{222} \mathrm{Ra}$ & ${ }^{14} \mathrm{C}$ & ${ }^{208} \mathrm{~Pb}$ & 8.90 & 11.01 & {$[2]$} \\
& & & & 11.09 & {$[4]$} \\
& & & & 11.22 & {$[13]$} \\
\hline${ }^{224} \mathrm{Ra}$ & ${ }^{14} \mathrm{C}$ & ${ }^{210} \mathrm{~Pb}$ & \multirow{2}{*}{15.06} & 15.86 & {$[2]$} \\
& & & & 15.68 & {$[15]$} \\
\hline${ }^{226} \mathrm{Ra}$ & ${ }^{14} \mathrm{C}$ & ${ }^{212} \mathrm{~Pb}$ & \multirow{2}{*}{18.98} & 21.19 & {$[4]$} \\
& & & & 21.24 & {$[5]$} \\
& & & & 21.34 & {$[11]$} \\
\hline
\end{tabular}

\begin{tabular}{lcccc}
\hline${ }^{226} \mathrm{Ra}$ & ${ }^{20} \mathrm{O}$ & ${ }^{206} \mathrm{Hg}$ & 23.53 & - \\
\hline \hline${ }^{226} \mathrm{Th}$ & ${ }^{18} \mathrm{O}$ & ${ }^{208} \mathrm{~Pb}$ & 17.31 & $>16.76$
\end{tabular}

\begin{tabular}{lccccc}
\hline${ }^{226} \mathrm{Th}$ & ${ }^{18} \mathrm{O}$ & ${ }^{208} \mathrm{~Pb}$ & 17.31 & $>16.76$ & {$[21]$} \\
\hline${ }^{228} \mathrm{Th}$ & ${ }^{20} \mathrm{O}$ & ${ }^{208} \mathrm{~Pb}$ & 19.53 & 20.72 & {$[16]$} \\
\hline
\end{tabular}

\begin{tabular}{llllll}
\hline${ }^{230} \mathrm{Th}$ & ${ }^{22} \mathrm{O}$ & ${ }^{208} \mathrm{~Pb}$ & 27.30 & - & \\
\hline${ }^{230} \mathrm{Th}$ & ${ }^{24} \mathrm{Ne}$ & ${ }^{206} \mathrm{Hg}$ & 25.08 & 24.60 & [22]
\end{tabular}

\begin{tabular}{lccccc}
\hline${ }^{232} \mathrm{Th}$ & ${ }^{22} \mathrm{O}$ & ${ }^{210} \mathrm{~Pb}$ & 32.25 & - & \\
\hline${ }^{232} \mathrm{Th}$ & ${ }^{24} \mathrm{Ne}$ & ${ }^{208} \mathrm{Hg}$ & 30.00 & $>29.20$ & {$[19]$} \\
${ }^{232} \mathrm{Th}$ & ${ }^{26} \mathrm{Ne}$ & ${ }^{206} \mathrm{Hg}$ & 29.65 & & \\
\hline
\end{tabular}

\begin{tabular}{lccccc}
\hline \hline${ }^{230} \mathrm{U}$ & ${ }^{20} \mathrm{O}$ & ${ }^{210} \mathrm{Po}$ & 25.67 & - & \\
\hline${ }^{230} \mathrm{U}$ & ${ }^{22} \mathrm{Ne}$ & ${ }^{208} \mathrm{~Pb}$ & 20.49 & 19.57 & {$[21]$} \\
\hline${ }^{232} \mathrm{U}$ & ${ }^{24} \mathrm{Ne}$ & ${ }^{208} \mathrm{~Pb}$ & 23.35 & 21.04 & {$[3]$} \\
& & & & 20.40 & {$[12]$} \\
& & & & 20.39 & {$[14]$} \\
\hline${ }^{234} \mathrm{U}$ & ${ }^{24} \mathrm{Ne}$ & ${ }^{210} \mathrm{~Pb}$ & 27.24 & 25.07 & {$[6]$} \\
${ }^{234} \mathrm{U}$ & ${ }^{26} \mathrm{Ne}$ & ${ }^{208} \mathrm{~Pb}$ & 28.02 & 25.25 & {$[8]$} \\
& & & & 25.30 & {$[9]$} \\
& & & & 25.93 & {$[14]$} \\
& & & & 25.89 & {$[14]$}
\end{tabular}

\begin{tabular}{|c|c|c|c|c|c|}
\hline${ }^{234} \mathrm{U}$ & ${ }^{28} \mathrm{Mg}$ & ${ }^{206} \mathrm{Hg}$ & 25.85 & $\begin{array}{l}25.54 \\
25.75 \\
25.54 \\
25.75\end{array}$ & $\begin{array}{c}{[6]} \\
{[8]} \\
{[9]} \\
{[14]}\end{array}$ \\
\hline $\begin{array}{l}{ }^{236} U \\
{ }^{236} U\end{array}$ & $\begin{array}{l}{ }^{24} \mathrm{Ne} \\
{ }^{26} \mathrm{Ne}\end{array}$ & $\begin{array}{l}{ }^{212} \mathrm{~Pb} \\
{ }^{210} \mathrm{~Pb}\end{array}$ & $\begin{array}{l}34.89 \\
35.49\end{array}$ & $>26.28$ & [9] \\
\hline${ }^{236} \mathrm{U}$ & $\begin{array}{l}{ }^{28} \mathrm{Mg} \\
{ }^{30} \mathrm{Mg}\end{array}$ & $\begin{array}{l}{ }^{208} \mathrm{Hg} \\
{ }^{206} \mathrm{Hg}\end{array}$ & $\begin{array}{l}33.68 \\
33.10\end{array}$ & $\begin{array}{c}>26.28 \\
27.58\end{array}$ & $\begin{array}{c}\text { [9] } \\
{[17]}\end{array}$ \\
\hline${ }^{236} \mathrm{Pu}$ & ${ }^{28} \mathrm{Mg}$ & ${ }^{208} \mathrm{~Pb}$ & 20.13 & $\begin{array}{l}21.67 \\
21.52\end{array}$ & $\begin{array}{l}{[10]} \\
{[18]}\end{array}$ \\
\hline
\end{tabular}

TABLE I. (Continued.)

\begin{tabular}{|c|c|c|c|c|c|}
\hline Emitter & Cluster & Residue & $\log \left(t_{1 / 2}^{\mathrm{HFB}}[\mathrm{s}]\right)$ & $\log \left(t_{1 / 2}^{\exp }[\mathrm{s}]\right)$ & Ref. \\
\hline${ }^{238} \mathrm{Pu}$ & ${ }^{28} \mathrm{Mg}$ & ${ }^{210} \mathrm{~Pb}$ & 29.42 & 25.70 & [7] \\
\hline${ }^{238} \mathrm{Pu}$ & ${ }^{30} \mathrm{Mg}$ & ${ }^{208} \mathrm{~Pb}$ & 29.52 & & \\
\hline${ }^{238} \mathrm{Pu}$ & ${ }^{32} \mathrm{Si}$ & ${ }^{206} \mathrm{Hg}$ & 28.23 & 25.27 & [7] \\
\hline${ }^{240} \mathrm{Pu}$ & ${ }^{34} \mathrm{Si}$ & ${ }^{206} \mathrm{Hg}$ & 26.96 & $>25.52$ & [22] \\
\hline${ }^{242} \mathrm{Cm}$ & ${ }^{34} \mathrm{Si}$ & ${ }^{208} \mathrm{~Pb}$ & 24.90 & 23.15 & [20] \\
\hline
\end{tabular}

observed cluster ${ }^{24} \mathrm{Ne}$ decreases faster than that for ${ }^{22} \mathrm{O}$ calculated in the "fusion" path. This produces a decrease of the width and the height of the barrier and increases the tunneling probability.

\section{CONCLUSIONS}

Cluster radioactivity is a very exotic kind of nuclear decay. It represents a bridge connecting the standard spontaneous nuclear fission phenomenon with $\alpha$ particle emission. We have shown here that this process can be understood as a kind of hyper-asymmetric spontaneous fission where the dynamics is governed by the shape of the potential energy surface given as a function of the quadrupole and octupole moments and computed in a fully microscopic way. The valley on the potential energy surface going through reflection-asymmetric shapes has been identified as responsible for the decay branch with emission of light cluster. The very high barriers involved in this process, reaching $25 \mathrm{MeV}$, result in extremely long halflives for this decay. The scission point of hyper-asymmetric fission is localized in the region of the saddle. The cluster is formed in the process of deforming a nucleus to a molecular shape with a heavy fragment close to the ${ }^{208} \mathrm{~Pb}$ nucleus. This approach is successful in predicting the mass and charge of the emitted particles as well as half-lives in each of the isotopes where the process is experimentally known.

The standard method for calculating the potential energy surface in the fully microscopic mean-field model and determining the fission paths has been applied in this paper. The innovation of our description as compared to standard fission is the use of the octupole moment as the leading coordinate driving the system from its ground-state configuration to hyper-asymmetric fission. This choice allows us to determine easily the fission path at the bottom of the valley and can be used in calculating half-lives.

A detailed analysis of the scission point has been performed. We have found the continuous path for the transition from a compound nucleus to two separated fragments. Applying constraints on the neck parameter is crucial in describing the potential energy surface in the region around the scission point without losing continuity. It has been proven that the entire up-going part of the fission path contributes to the energy barrier. At its end a neck is ruptured without change of the quadrupole or octupole moment, but with a rapid decrease 
of energy. The rest of the barrier describing the energy of the separated fragments can be approximated by the classical expression for the Coulomb energy between two charged spheres.

\section{ACKNOWLEDGMENTS}

The work of LMR was supported by Ministerio de Ciencia e Innovación (Spain) Grants No. FPA2009-08958 and No. FIS2009-07277, as well as by Consolider-Ingenio 2010 Programs CPAN CSD2007-00042 and MULTIDARK CSD200900064. The work of MW was supported by Ministerstwo Nauki i Szkolnictwa Wyższego (Poland) under Grant No. N N202 231137. M.W. is grateful to M. Kowal for stimulating discussions.

\section{APPENDIX A: CONVERGENCE OF THE ENERGY WITH BASIS SIZE}

The axially symmetric harmonic oscillator (HO) basis used to expand the HFB quasiparticle operators is characterized by the number of shells chosen for the $z$ and perpendicular directions and by the corresponding oscillator lengths $b_{z}$ and $b_{\perp}$. The number of shells is restricted by the condition $\frac{1}{q} n_{z}+\left(2 n_{\perp}+|m|\right) \leqslant N$, where $q$ and $N$ are parameters characterizing the basis. Along the perpendicular direction we take $N$ shells, (i.e., $2 n_{\perp}+|m|=0, \ldots, N$ ) and along the $z$ direction we include up to $q N$ shells depending on the value of $2 n_{\perp}+|m|$. In our calculations we have taken $q=1.5$, which is a good choice for elongated matter distributions extending along the $z$ direction, and for $N$ (which determines roughly the number of shells present in the basis) we have taken the values $11,13,15$, and 17 in order to check the convergence of the calculations with the basis size.

In all cases the oscillator lengths $b_{z}$ and $b_{\perp}$ characterizing the basis are optimized to minimize the energy for each value of the considered octupole moment. The results of the check for the nucleus ${ }^{238} \mathrm{Pu}$ along the $\mathrm{CR}$ path are summarized in Fig. 14. In this figure we have plotted the HFB energy as a function of $Q_{3}$ for all the values of $N$ used. We can see that as $N$ increases, the basis size increases and the energy (a variational quantity) decreases. The change in energy in going from $N$ to $N+2$ (as seen in the picture) decreases with increasing $N$. This change increases with the quadrupole moment, suggesting that very elongated shapes require a bigger basis for a proper description. We clearly observe a nice convergence of the energy as $N$ increases, giving us confidence that the $N=17$ results are already a faithful image of results to be obtained with an infinite basis. However, this basis is still rather demanding of long computational time (with CPU time being usually multiplied by a factor of 8 when $N$ is doubled) for the extensive calculations considered in this paper and we have taken $N=15$ in all the calculations considered in the paper. This choice provides energies which are roughly parallel to the $N=17$ values for octupole moments up to $Q_{3}=80 \mathrm{~b}^{3 / 2}$. The difference between $N=15$ and $N=17$ changes only from $0.6 \mathrm{MeV}$ for $Q_{3}=0 \mathrm{~b}^{3 / 2}$ to $1.6 \mathrm{MeV}$ for $Q_{3}=60 \mathrm{~b}^{3 / 2}$ and

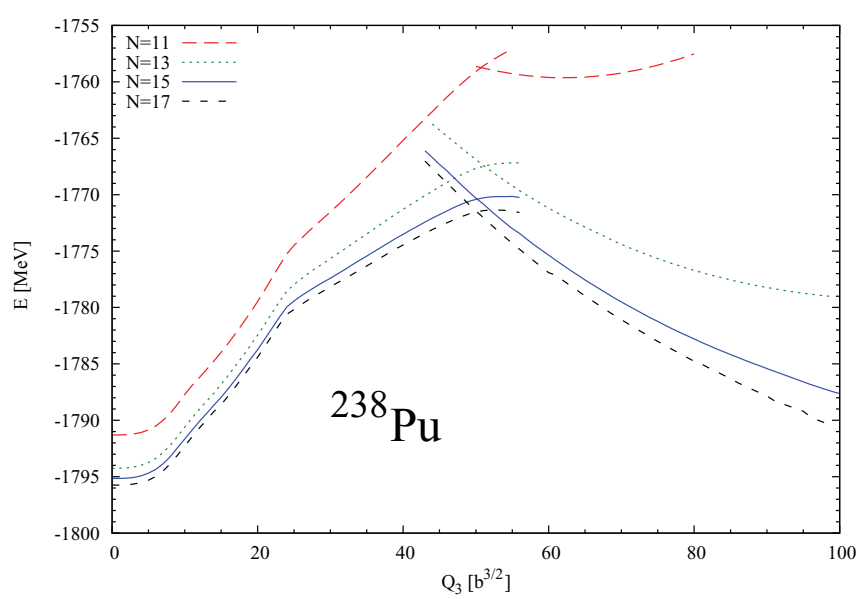

FIG. 14. (Color online) Convergence of the computed energy with the basis size characterized by the basis size parameter $N$ (see text for details).

2.0 MeV for $Q_{3}=80 \mathrm{~b}^{3 / 2}$. Therefore, the results depending upon energy differences will not be affected by our choice. However, for octupole moments greater than $Q_{3}=80 \mathrm{~b}^{3 / 2}$ the $N=15$ and $N=17$ bases are no longer parallel (with the difference between energies for $Q_{3}=80 \mathrm{~b}^{3 / 2}$ and $Q_{3}=$ $100 \mathrm{~b}^{3 / 2}$ growing by $1 \mathrm{MeV}$ ), suggesting poor convergence of the $N=15$ basis. Tests for bases beyond $N=17$ are hard to perform as very large bases show numerical instabilities in the evaluation of matrix elements of the interaction due to the finite accuracy of the computer's floating-point arithmetic. As discussed in the body of the paper, this region corresponds to two separate fragments where only the Coulomb repulsion energy changes with $Q_{3}$ and in this case it is better to use the classical expression for the Coulomb energy.

Despite the too-high energy of the cluster radioactivity valley for $N=13$ its shape is already well defined. Both "fission" and "fusion" paths have shapes that match exactly the ones of the larger bases. The cluster radioactivity phenomenon can be qualitatively explained in this case although some quantities (barrier heights and half-lives) will be overestimated.

\section{APPENDIX B: VARIOUS GOGNY FORCES}

Recently, two new parametrizations of the Gogny force have been presented: D1N [88] and D1M [89]. The parameters of D1N were constrained to reproduce, in addition to the standard requirements, the shape of a realistic symmetric and neutron matter equations of state. The idea was to improve the properties of the force for neutron-rich nuclei. The D1M parametrization also included the requirements of $\mathrm{D} 1 \mathrm{~N}$ but the parameters were fitted to the binding energies of the whole even-even nuclide chart and therefore its binding energy rms is outstanding. The properties of D1M have been tested in several scenarios $[100,101]$ with very promising results. 


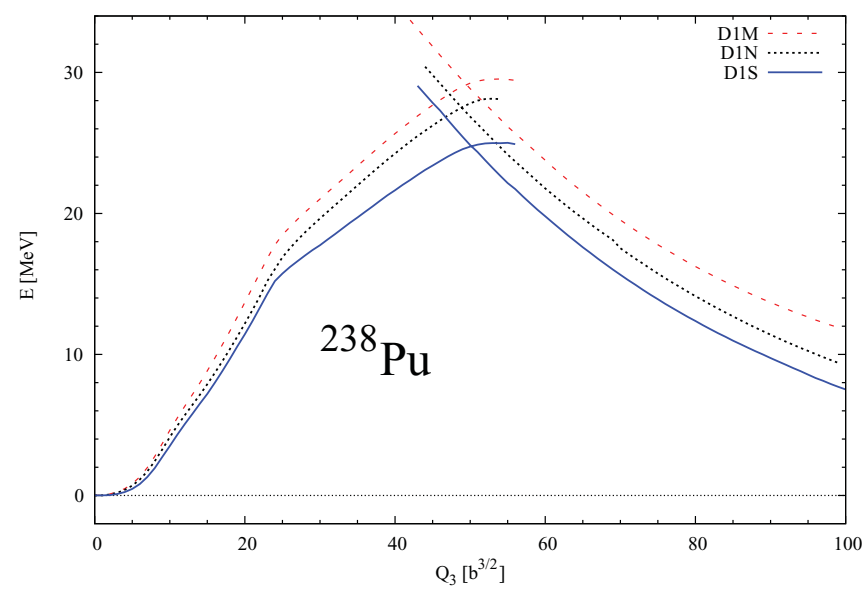

FIG. 15. (Color online) The hyper-asymmetric barrier in ${ }^{238} \mathrm{Pu}$ calculated with different parametrizations of the Gogny force: D1S, D1N, and D1M.
It is interesting to test the performance of the three parametrizations in the very demanding scenario of $\mathrm{CR}$ and to this end we have plotted in Fig. 15 the results for the PES of CR for the nucleus ${ }^{238} \mathrm{Pu}$ obtained with the three parameterizations. The shape of the PES is similar in the three cases, although the hyper-asymmetric fission barrier is $2 \mathrm{MeV}$ higher in D1N and $3 \mathrm{MeV}$ in D1M than in D1S. The predicted cluster is the same ${ }^{30} \mathrm{Mg}$ nucleus in all three parametrizations. The changes of the barrier heights lead to an increase of the CR half-lives. For the D1M parameter set in the case of emission of ${ }^{30} \mathrm{Mg}$ from ${ }^{238} \mathrm{Pu}$ we get $\log \left(t_{1 / 2}[\mathrm{~s}]\right)=$ 34.33 (without ZPE correction added) and for the D1N force $\log \left(t_{1 / 2}[\mathrm{~s}]\right)=32.28$ in comparison with $\log \left(t_{1 / 2}[\mathrm{~s}]\right)=30.42$ for the D1S parametrization.

The new Gogny parametrizations D1M and D1N provide a similar picture of the $\mathrm{CR}$ phenomenon, although numerical calculations give slightly larger values of barrier heights and half-lives.
[1] H. J. Rose and G. A. Jones, Nature (London) 307, 245 (1984).

[2] P. B. Price, J. D. Stevenson, S. W. Barwick, and H. L. Ravn, Phys. Rev. Lett. 54, 297 (1985).

[3] S. W. Barwick, P. B. Price, and J. D. Stevenson, Phys. Rev. C 31, 1984 (1985).

[4] E. Hourani, M. Hussonnois, L. Stab, L. Brillard, S. Gales, and J. P. Schapira, Phys. Lett. B 160, 375 (1985).

[5] S. W. Barwick, P. B. Price, H. L. Ravn, E. Hourani, and M. Hussonnois, Phys. Rev. C 34, 362 (1986).

[6] S. Wang, P. B. Price, S. W. Barwick, K. J. Moody, and E. K. Hulet, Phys. Rev. C 36, 2717 (1987).

[7] S. Wang, D. Snowden-Ifft, P. B. Price, K. J. Moody, and E. K. Hulet, Phys. Rev. C 39, 1647 (1989).

[8] K. J. Moody, E. K. Hulet, S. Wang, and P. B. Price, Phys. Rev. C 39, 2445 (1989).

[9] S. P. Tretyakova, Yu. S. Zamyatnin, V. N. Kovantsev, Yu. S. Korotkin, V. L. Mikheev, and G. A. Timofeev, Z. Phys. A 333, 349 (1989).

[10] A. A. Ogloblin et al., Phys. Lett. B 235, 35 (1990).

[11] D. Weselka, P. Hille, and A. Chalupka, Phys. Rev. C 41, 778 (1990).

[12] R. Bonetti, E. Fioretto, C. Migliorino, A. Pasinetti, F. Barranco, E. Vigezzi, and R. A. Broglia, Phys. Lett. B 241, 179 (1990).

[13] M. Hussonnois, J. F. Le Du, L. Brillard, J. Dalmasso, and G. Ardisson, Phys. Rev. C 43, 2599 (1991).

[14] R. Bonetti, C. Chiesa, A. Guglielmetti, C. Migliorino, A. Cesana, M. Terrani, and P. B. Price, Phys. Rev. C 44, 888 (1991).

[15] E. Hourani et al., Phys. Rev. C 44, 1424 (1991).

[16] R. Bonetti, C. Chiesa, A. Guglielmetti, C. Migliorino, A. Cesana, and M. Terrani, Nucl. Phys. A 556, 115 (1993).

[17] S. P. Tret'yakova, V. L. Mikheev, V. A. Ponomarenko, A. N. Golovchenko, A. A. Ogloblin, and V. A. Shigin, JETP Lett. 59, 394 (1994).

[18] M. Hussonnois et al., JETP Lett. 62, 701 (1995).

[19] R. Bonetti, C. Chiesa, A. Guglielmetti, R. Matheoud, G. Poli, V. L. Mikheev, and S. P. Tretyakova, Phys. Rev. C 51, 2530 (1995).
[20] A. A. Ogloblin et al., Phys. Rev. C 61, 034301 (2000).

[21] R. Bonetti, C. Carbonini, A. Guglielmetti, M. Hussonnois, D. Trubert, and C. Le Naour, Nucl. Phys. A 686, 64 (2001).

[22] R. Bonetti and A. Guglielmetti, in Heavy Elements and Related Phenomena, Vol. II, edited by W. Greiner and R. K. Gupta (World Scientific, Singapore, 1999), p. 643.

[23] D. N. Poenaru, Y. Nagame, R. A. Gherghescu, and W. Greiner, Phys. Rev. C 65, 054308 (2002); 66, 049902(E) (2002).

[24] Yu. Ts. Oganessian, Yu. A. Lazarev, V. L. Mikheev, Yu. A. Muzychka, I. V. Shirokovsky, S. P. Tretyakova, and V. K. Utyonkov, Z. Phys. A 349, 341 (1994).

[25] A. Guglielmetti et al., Phys. Rev. C 52, 740 (1995).

[26] A. Guglielmetti et al., Phys. Rev. C 56, R2912 (1997).

[27] G. Audi, O. Bersillon, J. Blachot, and A. H. Wapstra, Nucl. Phys. A 729, 3 (2003).

[28] O. Hahn and F. Strassmann, Naturwissenschaften 27, 11 (1939).

[29] S. P. Tretyakova, A. A. Ogloblin, and G. A. Pik-Pichak, Phys. Atom. Nucl. 66, 1618 (2003).

[30] A. Sandulescu, D. N. Poenaru, and W. Greiner, Sov. J. Part Nucl. 11, 528 (1980).

[31] J. L. Egido and L. M. Robledo, Nucl. Phys. A 738, 31 (2004).

[32] L. M. Robledo and M. Warda, Int. J. Mod. Phys. E 17, 204 (2008).

[33] L. M. Robledo and M. Warda, Int. J. Mod. Phys. E 17, 2275 (2008).

[34] M. Warda, Acta Phys. Pol. B 42, 477 (2011).

[35] B. Buck and A. C. Merchant, J. Phys. G 16, L85 (1990).

[36] R. Blendowske, T. Fliessbach, and H. Walliser, Z. Phys. A 339, 121 (1991).

[37] D. N. Poenaru, D. Schnabel, W. Greiner, D. Mazilu, and R. Gherghescu, At. Data Nucl. Data Tables 48, 231 (1991).

[38] D. N. Poenaru and W. Greiner, Phys. Scr. 44, 427 (1991).

[39] R. K. Gupta and W. Greiner, Int. J. Mod. Phys. E 3, 335 (1994).

[40] S. Kumar and R. K. Gupta, Phys. Rev. C 55, 218 (1997).

[41] G. Royer and R. K. Gupta, and V. Yu. Denisov, Nucl. Phys. A 632, 275 (1998). 
[42] B. Buck, A. C. Merchant, and S. M. Perez, Nucl. Phys. A 657, 267 (1999).

[43] C. Mazzocchi, A. Guglielmetti, R. Bonetti, and R. K. Gupta, Phys. Rev. C 61, 047304 (2000).

[44] G. Royer and R. Moustabchir, Nucl. Phys. A 683, 182 (2001).

[45] D. N. Basu, Phys. Rev. C 66, 027601 (2002).

[46] M. Balasubramaniam, S. Kumarasamy, N. Arunachalam, and R. K. Gupta, Phys. Rev. C 70, 017301 (2004).

[47] M. Horoi, J. Phys. G 30, 945 (2004).

[48] A. Iwamoto, T. Ichikawa, P. Moller, and A. J. Sierk, Nucl. Phys. A 738, 499 (2004).

[49] A. A. Ogloblin, S. P. Trctyakov, R. N. Sagaidak, S. A. Goncharov, and G. A. Pik-Pichak, Nucl. Phys. A 738, 313 (2004)

[50] O. A. P. Tavares, L. A. M. Roberto, and E. L. Medeiros, Phys. Scr. 76, 375 (2007).

[51] D. Ni, Z. Ren, T. Dong, and C. Xu, Phys. Rev. C 78, 044310 (2008)

[52] C. Qi, F. R. Xu, R. J. Liotta, R. Wyss, M. Y. Zhang, C. Asawatangtrakuldee, and D. Hu, Phys. Rev. C 80, 044326 (2009).

[53] T. R. Routray, J. Nayak, and D. N. Basu, Nucl. Phys. A 826, 223 (2009).

[54] D. N. Poenaru and W.Greiner, Nucl. Phys. A 834, 163c (2010).

[55] K. P. Santhosh, R. K. Biju, and S. Sahadevan, Nucl. Phys. A 838, 38 (2010).

[56] B. Singh, S. K. Patra, and R. K. Gupta, Phys. Rev. C 82, 014607 (2010).

[57] D. N. Poenaru, R. A. Gherghescu, and W. Greiner, Phys. Rev. C 83, 014601 (2011).

[58] Z. Sheng, D. Ni, and Z. Ren, J. Phys. G 38, 055103 (2011).

[59] G. Shivakumaraswamy and T. K. Umesh, Phys. At. Nucl. 74, 675 (2011).

[60] D. N. Poenaru (Ed.), Nulcear Decay Modes (Institute of Physics, Bristol, 1996).

[61] M. A. Hooshyar, I. Reichstein, and F. B. Malik, Nuclear Fission and Cluster Radioactivity: An Energy-Density Functional Approach (Springer-Verlag, Berlin, 2005).

[62] Ch. Beck (Ed.), Clusters in Nuclei, Volume 1, Lecture Notes in Physics Vol. 818 (Springer, Heidelberg, 2010).

[63] Ch. Beck (Ed.), Clusters in Nuclei, Volume 2, Lecture Notes in Physics (Springer, Heidelberg, 2012).

[64] D. Ni and Z. Ren, Phys. Rev. C 82, 024311 (2010).

[65] B. Singh, S. K. Patra, and R. K. Gupta, Phys. Rev. C 82, 014607 (2010).

[66] J. M. Dong, H. F. Zhang, J. Q. Li, and W. Scheid, Eur. Phys. J. A 41, 197 (2009).

[67] M. Mirea, Phys. Rev. C 78, 044618 (2008).

[68] D. N. Poenaru, R. A. Gherghescu, and W. Greiner, Phys. Rev. C 73, 014608 (2006).

[69] M. Warda, J. L. Egido, L. M. Robledo, and K. Pomorski, Phys. Rev. C 66, 014310 (2002).

[70] M. Warda, K. Pomorski, J. L. Egido, and L. M. Robledo, Int. J. Mod. Phys. E 14, 403 (2005).

[71] M. Warda, K. Pomorski, J. L. Egido, and L. M. Robledo, J. Phys. G 31, S1555 (2005).
[72] M. Warda, Eur. Phys. J. A 42, 605 (2009).

[73] J. F. Berger, M. Girod, and D. Gogny, Nucl. Phys. A 428, 23c (1984).

[74] J.-F. Berger, J. D. Anderson, P. Bonche, and M. S. Weiss, Phys. Rev. C 41, R2483 (1990).

[75] J.-F. Berger, M. Girod, and D. Gogny, Comput. Phys. Commun. 63, 365 (1991).

[76] J.-P. Delaroche, M. Girod, H. Goutte, and J. Libert, Nucl. Phys. A 771, 103 (2006).

[77] N. Dubray, H. Goutte, and J.-P. Delaroche, Phys. Rev. C 77, 014310 (2008).

[78] J. Dechargé and D. Gogny, Phys. Rev. C 21, 1568 (1980).

[79] P. Ring and P. Shuck, The Nuclear Many Body Problem (Springer-Verlag, Berlin, 1980).

[80] J. L. Egido, J. Lessing, V. Martin, and L. M. Robledo, Nucl. Phys. A 594, 70 (1995).

[81] L. M. Robledo and G. F. Bertsch, Phys. Rev. C 84, 014312 (2011).

[82] V. Martin and L. M. Robledo, Int. J. Mod. Phys. E 18, 788 (2009).

[83] S. Perez-Martin and L. M. Robledo, Int. J. Mod. Phys. E 18, 861 (2009).

[84] W. Younes and D. Gogny, Phys. Rev. C 80, 054313 (2009).

[85] J.-F. Berger, M. Girod, and D. Gogny, Nucl. Phys. A 502, 85 (1989).

[86] J. L. Egido and L. M. Robledo, Phys. Rev. Lett. 85, 1198 (2000).

[87] J.-F. Berger and K. Pomorski, Phys. Rev. Lett. 85, 30 (2000).

[88] F. Chappert, M. Girod, and S. Hilaire, Phys. Lett. B 668, 420 (2008).

[89] S. Goriely, N. Chamel, and J. M. Pearson, Phys. Rev. Lett. 102, 152503 (2009).

[90] R. Rodríguez-Guzmán, J. L. Egido, and L. M. Robledo, Phys. Lett. B 474, 15 (2000).

[91] R. Rodríguez-Guzmán, J. L. Egido, and L. M. Robledo, Nucl. Phys. A 709, 201 (2002).

[92] J. L. Egido and L. M. Robledo, Lect. Notes Phys. 641, 269 (2004).

[93] M. Girod, J. P. Delaroche, J. Libert, and I. Deloncle, Phys. Rev. C 45, R1420 (1992).

[94] M. J. Giannoni and P. Quentin, Phys. Rev. C 21, 2060 (1980); 21, 2076 (1980).

[95] D. L. Hill and J. A. Wheeler, Phys. Rev. 89, 1102 (1953).

[96] K. Pomorski, Int. J. Mod. Phys. E 17, 245 (2008).

[97] G. Audi, A. H. Wapstra, and C. Thibault, Nucl. Phys. A 729, 337 (2003).

[98] P. Moller, J. R. Nix, and W. J. Swiatecki, At. Data Nucl. Data Tables 59, 185 (1995).

[99] [http://www-phynu.cea.fr/science_en_ligne/carte_potentiels_ microscopiques/carte_potentiel_nucleaire.htm].

[100] R. Rodriguez-Guzman, P. Sarriguren, L. M. Robledo, and S. Perez-Martin, Phys. Lett. B 691, 202 (2010); R. RodriguezGuzman, P. Sarriguren, and L. M. Robledo, Phys. Rev. C 82, 061302(R) (2010).

[101] M. Warda and A. Staszczak, and L. Próchniak, Int. J. Mod. Phys. E 19, 787 (2010). 